\title{
Rhodium-Catalyzed One-Pot Synthesis of Substituted Pyridine Derivatives from $\alpha, \beta$-Unsaturated Ketoximes and Alkynes
}

Kanniyappan Parthasarathy, Masilamani Jeganmohan and Chien-Hong Cheng*

Department of Chemistry, National Tsing Hua University, Hsinchu 30013, Taiwan

\section{Supporting Information}

\section{Table of Contents}

S2 Experimental Section

S3 - S10 Spectral Data of all Compounds

S10-11 NOE data

S11 References

S12 - $30 \quad{ }^{1} \mathrm{H}$ and ${ }^{13} \mathrm{C}$ NMR Spectra of All Compounds 


\section{Experimental Section}

General. All reactions were conducted under nitrogen atmosphere on a dual-manifold Schlenk line unless otherwise mentioned and in oven-dried glass wares. All solvents were dried according to known methods and distilled prior to use. ${ }^{1}$ Starting materials substituted ketoximes $\mathbf{1} \mathbf{a}-\mathbf{i},{ }^{2}$ and symmetrical alkyne $\mathbf{2} \mathbf{b}^{3}$ were synthesized according to the literature procedures. Chlorotris(triphenylphosphine)rhodium $\left(\mathrm{RhCl}\left(\mathrm{PPh}_{3}\right)_{3}\right.$, Wilkinson catalyst) was prepared according to the literature procedure and stored in a refrigerator under $\mathrm{N}_{2}$ atmosphere. ${ }^{4}$ Other reagents were commercially available and used as purchased.

\section{Optimization Studies for the Reaction of $\alpha, \beta$-Unsaturated Ketoximes with}

Alkynes $^{\mathrm{a}}$

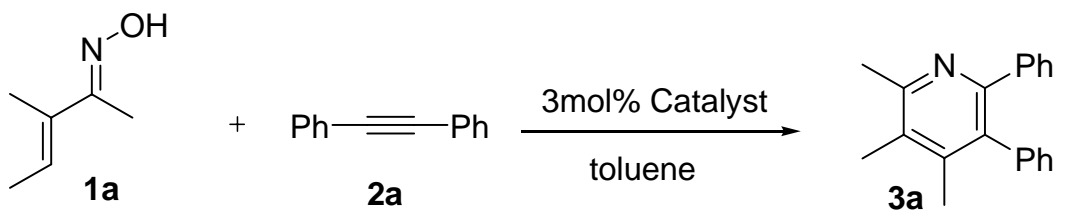

\begin{tabular}{|c|c|c|c|c|}
\hline Entry & Catalyst & Temp $\left({ }^{\circ} \mathrm{C}\right)$ & Time(h) & Yield (\%) \\
\hline 1 & No & 150 & 15 & 0 \\
\hline 2 & {$[\mathrm{RhCl}(\mathrm{COD})]_{2}$} & 130 & 12 & 0 \\
\hline 3 & {$[\mathrm{RhCl}(\mathrm{COD})]_{2} / 4 \mathrm{P}(\mathrm{Cy})_{3}$} & 130 & 12 & 35 \\
\hline 4 & {$[\mathrm{RhCl}(\mathrm{COD})]_{2} / 4 \mathrm{PPh}_{3}$} & 130 & 12 & 56 \\
\hline 5 & {$[\mathrm{RhCl}(\mathrm{COD})]_{2} / 2 \mathrm{bPy}$} & 130 & 12 & 0 \\
\hline 6 & $\mathrm{RhCl}\left(\mathrm{PPh}_{3}\right)_{3}$ & 130 & 12 & 92 \\
\hline 7 & $\mathrm{RhCl}\left(\mathrm{PPh}_{3}\right)_{3}$ & 130 & 3 & 92 \\
\hline
\end{tabular}

${ }^{\mathrm{a}}$ All reactions were carried out using $\alpha, \beta$-unsaturated ketoxime 1a (1.00 mmol), diphenylacetylene $2 \mathbf{a}(1.1 \mathrm{mmol})$ and catalyst $(3 \mathrm{~mol} \%)$ in the presence of toluene (2.0 mL). ${ }^{\text {b}}$ Yields were measured from crude products by the ${ }^{1} \mathrm{H}$ NMR integration method using mesitylene as an internal standard. 
General procedure for the synthesis of substituted pyridine derivatives. A sealed tube containing $\mathrm{RhCl}\left(\mathrm{PPh}_{3}\right)_{3}(0.030 \mathrm{mmol}, 3.0 \mathrm{~mol} \%)$ was evacuated and purged with nitrogen gas three times. Freshly distilled toluene $(2.0 \mathrm{~mL})$, oxime $(1.00$ mmol) and alkyne $(1.10 \mathrm{mmol})$ were sequentially added to the system and the reaction mixture was allowed to stir at $130{ }^{\circ} \mathrm{C}$ for $3 \mathrm{~h}$. The mixture was filtered through a short Celite pad and washed with dichloromethane several times. The filtrate was concentrated and the residue was purified on a neutral silica gel column using hexanes-ethyl acetate as eluent to afford the substituted pyridine derivative $\mathbf{3}$.

Spectral data and copies of ${ }^{1} \mathrm{H}$ and ${ }^{13} \mathrm{C}$ NMR spectra of all compounds are listed below.

\section{2,3,4-Trimethyl-5,6-diphenylpyridine (3a).}

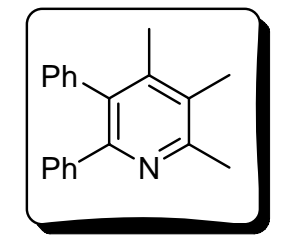

Yellow solid; m.p. 59-61 ${ }^{\circ} \mathrm{C} ;{ }^{1} \mathrm{H}$ NMR (400 MHz, $\mathrm{CDCl}_{3}$ ): $\delta 7.23$-7.71 (m, 5 H), 7.11 - 7.09 (m, 3 H), 7.02 -7.00 (m, 2 H), 2.62 (s, 3 H), 2.29 (s, 3 H), 2.07 (s, 3 H); ${ }^{13} \mathrm{C}$ NMR (100 MHz, $\left.\mathrm{CDCl}_{3}\right): \delta 159.4,154.0,144.2,141.2,139.3,133.8,130.6,129.7$, 128.8, 127.9, 127.4, 126.7, 126.6, 23.5, 17.5, 15.4; IR (KBr): 3054, 2923, 1453, 1550 $(\mathrm{C}=\mathrm{N}) \mathrm{cm}^{-1}$; HRMS calcd for $\mathrm{C}_{20} \mathrm{H}_{19} \mathrm{~N}$ 273.1517, found 273.1522.

4,6-Dimethyl-2,3-diphenylpyridine (3b).

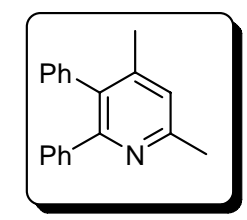

Pale yellow solid; m.p. $68-70{ }^{\circ} \mathrm{C} ;{ }^{1} \mathrm{H}$ NMR (400 MHz, $\left.\mathrm{CDCl}_{3}\right)$ : $\delta 7.25$ - 7.22 (m, 5 H), 7.14 - 7.13 (m, 3 H), 7.08 (s, 1 H), 7.07- 7.05 (m, 2 H), 2.62 (s,3 H), 2.14 (s, 3 $\mathrm{H}) ;{ }^{13} \mathrm{C}$ NMR (100 MHz, $\left.\mathrm{CDCl}_{3}\right): \delta 156.9,156.4,146.2,140.8,138.4,133.2,130.3$, 
129.7, 128.0, 127.4, 126.9, 126.7, 123.4, 24.2, 20.5; IR (KBr): 3062, 2958, 2923, $1596(\mathrm{C}=\mathrm{N}), 1488,1546, \mathrm{~cm}^{-1}$; HRMS calcd for $\mathrm{C}_{19} \mathrm{H}_{17} \mathrm{~N}$ 259.1361, found 259.1363.

\section{6-Ethyl-4-methyl-2,3-diphenylpyridine (3c).}

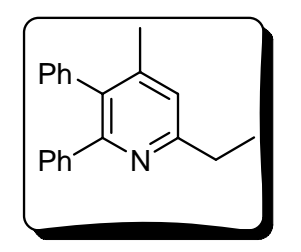

Yellow viscous oil; ${ }^{1} \mathrm{H}$ NMR (400 MHz, $\left.\mathrm{CDCl}_{3}\right): \delta 7.26$ - 7.25 (m, $\left.3 \mathrm{H}\right), 7.23$ 7.20 (m,2 H), 7.14 - 7.11 (m, 3H), 7.07 (s, 1 H), 7.06 - 7.03 (m, 2 H), 2.90 (q, J =7.2 Hz,2 H), 2.14 (s,3 H), 1.39 (t, $J=1.6$ Hz,3 H); ${ }^{13} \mathrm{C}$ NMR (100 MHz, $\left.\mathrm{CDCl}_{3}\right): \delta 161.7$, 156.8, 146.3, 140.50, 138.6, 133.4, 130.33, 129.8, 128.0, 127.4, 126.9, 126.7, 122.0, 31.17, 20.68, 14.09; IR (KBr): 3080, 2985, 2938, $1498(\mathrm{C}=\mathrm{N}) \mathrm{cm}^{-1}$; HRMS calcd for $\mathrm{C}_{20} \mathrm{H}_{19} \mathrm{~N}$ 273.1517, found 273.1520.

\section{6-Methyl-2,3-diphenylpyridine (3d).}

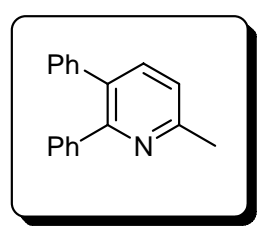

Yellow viscous oil; ${ }^{1} \mathrm{H}$ NMR (400 MHz, $\mathrm{CDCl}_{3}$ ): $\delta 7.60$ (d, $J=7.6 \mathrm{~Hz}, 1 \mathrm{H}$ ), 7.34 - 7.32 (m, $1 \mathrm{H}), 7.23$ - 7.12 (m, $10 \mathrm{H}), 2.65$ (s, $3 \mathrm{H}) ;{ }^{13} \mathrm{C}$ NMR (100 MHz, $\left.\mathrm{CDCl}_{3}\right): \delta 157.0,156.4,140.3,140.0,138.6,133.0,129.8,129.4,128.1,127.7,127.4$ 126.7, 121.6, 24.3; IR (KBr): 3074, 3049, 2954, $1576(\mathrm{C}=\mathrm{N}) \mathrm{cm}^{-1}$; HRMS calcd for $\mathrm{C}_{18} \mathrm{H}_{15} \mathrm{~N} 245.1204$, found 245.1207.

2,3-Diphenyl-6-propylpyridine (3e).

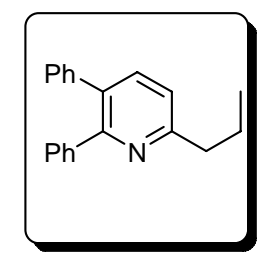


Pale yellow solid; m.p. $58-60{ }^{\circ} \mathrm{C} ;{ }^{1} \mathrm{H}$ NMR (400 MHz, $\left.\mathrm{CDCl}_{3}\right): \delta 7.63$ (d, $J=8.0$ Hz, 1 H), 7.36 - 7.31 (m, 3 H), $\delta 7.27$ - 7.14 (m, 8 H), 2.89 - 2.83 (quintet, 2 H), 1.88 1.80 (m, $2 \mathrm{H}), 1.05$ (t, $J=7.2, \mathrm{~Hz}, 3 \mathrm{H}) ;{ }^{13} \mathrm{C} \mathrm{NMR}$ (100 MHz, $\left.\mathrm{CDCl}_{3}\right): \delta$ 156.7, 156.4, 140.3, 139.9, 138.6, 132.9, 129.8, 129.4, 128.1, 127.7, 127.4, 126.7, 121.6, 24.3; IR (KBr): 3068, 3054, 3026, 2935, 2897, $1581(\mathrm{C}=\mathrm{N}) \mathrm{cm}^{-1}$; HRMS calcd for $\mathrm{C}_{20} \mathrm{H}_{19} \mathrm{~N}$ 273.1517, found 273.1521.

1-Methyl-3,4-diphenyl-6,7-dihydro-5H-cyclopenta[c]pyridine (3f).

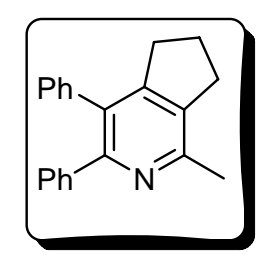

Yellow solid; m.p. $103-105{ }^{\circ} \mathrm{C} ;{ }^{1} \mathrm{H}$ NMR (400 MHz, $\left.\mathrm{CDCl}_{3}\right): \delta 7.49$ - 7.46 (m, 3 H), 7.42 - 7.22 (m, 2 H), 7.16 - 7.15 (m, 3 H), 7.08 -7.07, (m, 2 H), 3.01 (t, $J=7.6$, Hz, 2 H), 2.82 (t, $J=7.6$ Hz, 2 H), 2.57 (s, 3 H), 2.13-2.06 (m, 2 H); ${ }^{13} \mathrm{C}$ NMR (100 $\left.\mathrm{MHz}, \mathrm{CDCl}_{3}\right): \delta 154.5,153.0,152.1,140.6,138.5,136.5,130.2,129.8,129.7,127.8$ 127.4, 126.8, 126.5, 32.8, 30.9, 24.5, 22.1; IR (KBr): 3079, 2981, 2897, $1595(\mathrm{C}=\mathrm{N})$ $\mathrm{cm}^{-1}$; HRMS calcd for $\mathrm{C}_{21} \mathrm{H}_{19} \mathrm{~N} 285.1517$, found 285.1517 .

[2,2,1]-Bicyclic pyridine derivative (3g).

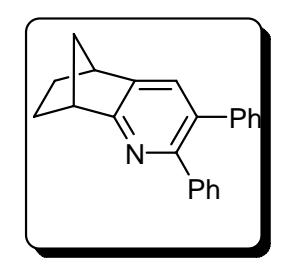

Yellow solid; m.p. $128-130{ }^{\circ} \mathrm{C} ;{ }^{1} \mathrm{H}$ NMR (400 MHz, $\left.\mathrm{CDCl}_{3}\right): \delta 7.43$ (s, $1 \mathrm{H}$ ), 7.33 - 7.29 (m, 2 H), 7.25 - 7.18 (m, 6 H), 7.17 - 7.13 (m, 2 H), 3.54 (s, 1 H), 3.48 (s, 1 H), 2.05-2.02 (m, 2 H), 1.96-1.93 (m, 1 H), 1.66-1.63 (m,1 H), 1.48-1.43 (m, 1 H), 1.35-1.25 (m, $1 \mathrm{H}) ;{ }^{13} \mathrm{C}$ NMR (100 MHz, $\left.\mathrm{CDCl}_{3}\right): \delta$ 167.8, 153.5, 140.9, 140.8, 139.7, 133.1, 130.1, 130.0, 129.7, 128.07, 127.7, 127.0, 126.5, 48.0, 45.13, 42.2, 27.0, 25.5; 
IR (KBr): 3054, 3031, 2962, 2869, $1553(\mathrm{C}=\mathrm{N}) \mathrm{cm}^{-1}$; HRMS calcd for $\mathrm{C}_{22} \mathrm{H}_{19} \mathrm{~N}$ 297.1517, found 297.1513.

1-Methyl-3,4-di(2-thienyl)-6,7-dihydro-5H-cyclopenta[c]pyridine(3h).

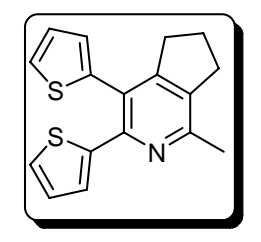

Yellow solid; m.p. $130-132{ }^{\circ} \mathrm{C} ;{ }^{1} \mathrm{H}$ NMR (400 MHz, $\left.\mathrm{CDCl}_{3}\right): \delta 7.40$ (d, $J=7.6$ Hz, $1 \mathrm{H}$ ), 7.22 (d, $J=8.0 \mathrm{~Hz}, 1 \mathrm{H}), 7.10-7.07$ (m, $1 \mathrm{H}$ ), 6.90 (d, $J=7.6, \mathrm{~Hz}, 1 \mathrm{H}$ ), 6.82-6.80 (m, $1 \mathrm{H}), 6.6$ (d, $J=7.6, \mathrm{~Hz}, 1 \mathrm{H}), 2.92$ (t, $J=7.2, \mathrm{~Hz}, 2 \mathrm{H}), 2.74$ (t, $J=7.2$, Hz, 2 H), 2.50 (s, 3 H), 2.10-2.00 (quintet, 2 H), 2.07 (s, 3 H); ${ }^{13} \mathrm{C}$ NMR (100 MHz, $\left.\mathrm{CDCl}_{3}\right): \delta 155.3,153.2,148.8,144.4,139.2,136.0,127.3,127.2,127.1,126.8,126.3$ 121.5, 32.7, 30.9, 24.0, 22.1; IR (KBr): 3093, 3062, 2976, 2943, $1573(\mathrm{C}=\mathrm{N}) \mathrm{cm}^{-1}$; HRMS calcd for $\mathrm{C}_{17} \mathrm{H}_{15} \mathrm{NS}_{2}$ 297.0646, found 297.0651.

2,3,4,5,6-Pentamethylpyridine (3i).

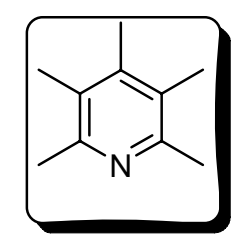

Pale yellow solid; m.p. $49-51{ }^{\circ} \mathrm{C} ;{ }^{1} \mathrm{H}$ NMR (400 MHz, $\mathrm{CDCl}_{3}$ ): $\delta 2.42$ (s, $6 \mathrm{H}, 2$ $\mathrm{CH}_{3}$ merged in same value), 2.15 (s, $6 \mathrm{H}, 2 \mathrm{CH}_{3}$ merged in same value), 2.14 (s, 3 $\mathrm{H}) ;{ }^{13} \mathrm{C}$ NMR (100 MHz, $\left.\mathrm{CDCl}_{3}\right): \delta$ 152.2, 143.6, 127.0, 23.1, 15.7, 15.1; IR: (KBr) 2992, 2923, 2869, 2730, $1586(\mathrm{C}=\mathrm{N}) \mathrm{cm}^{-1}$; HRMS calcd for $\mathrm{C}_{10} \mathrm{H}_{15} \mathrm{~N}$ 149.1204, found 149.1207.

2,3-Diethyl-6-methyl-4-phenylpyridine (3j).

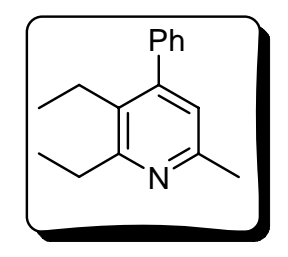


Brown liquid; ${ }^{1} \mathrm{H}$ NMR (400 MHz, $\mathrm{CDCl}_{3}$ ): $\delta 7.40$ - 7.25 (m, $3 \mathrm{H}$ ), 7.23 -7.22 (m, 2 H), 6.78 (s, 1 H), 2.86 (q, $J=7.6 \mathrm{~Hz}, 2 \mathrm{H}), 2.54$ (q, $J=7.6,2 \mathrm{H}), 2.49$ (s, 3 H),1.32 (t, $J=7.2, \mathrm{~Hz}, 3 \mathrm{H}), 0.98$ (t, $J=7.2, \mathrm{~Hz}, 3 \mathrm{H}) ;{ }^{13} \mathrm{C}$ NMR $\left(100 \mathrm{MHz}, \mathrm{CDCl}_{3}\right)$ : $\delta 161.2,154.2,150.3,140.4,130.9,128.3,128.0,127.3,122.0,28.3,23.9,21.4,15.3$, 14.6; IR (KBr): 3048, 3012, 2973, 2914, $28491530(\mathrm{C}=\mathrm{N}) \mathrm{cm}^{-1}$; HRMS calcd for $\mathrm{C}_{16} \mathrm{H}_{19} \mathrm{~N}$ 225.1517, found 225.1519.

\section{1-Methyl-3,4-dipropyl-5,6,7,8-tetrahydroisoquinoline (3k).}

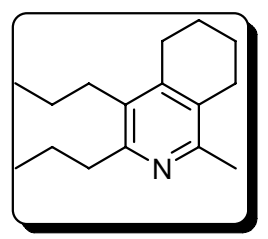

Yellow viscous oil; ${ }^{1} \mathrm{H}$ NMR (400 MHz, $\left.\mathrm{CDCl}_{3}\right)$ : $\delta 2.67$ - 2.63 (m, $\left.4 \mathrm{H}\right), 2.56$ 2.54 (m, 2 H), 2.50 - 2.46 (m, 2 H), 2.35 (s,3 H), 1.76 - 1.70 (m, 4 H), 1.68 - 1.62 (m, $2 \mathrm{H}), 1.47-1.41$ (m,2 H), 1.01 - 0.96 (m, $6 \mathrm{H}) ;{ }^{13} \mathrm{C}$ NMR (100 MHz, $\left.\mathrm{CDCl}_{3}\right): \delta$ 155.5, 153.2, 143.8, 130.7, 127.8, 37.2, 26.4, 23.8, 22.5, 22.4, 22.0, 14.6, 14.4; IR (KBr): 2984, 2936, 2848, $1491(\mathrm{C}=\mathrm{N}) \mathrm{cm}^{-1}$; HRMS calcd for $\mathrm{C}_{16} \mathrm{H}_{25} \mathrm{~N}$ 231.1987, found 231.1989.

\section{1-Methyl-4-phenyl-6,7-dihydro-5H-cyclopenta[c]pyridine (3l).}

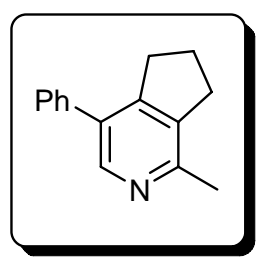

Brown color viscous oil; ${ }^{1} \mathrm{H}$ NMR (400 MHz, $\mathrm{CDCl}_{3}$ ): $\delta 8.30$ (s, $\left.1 \mathrm{H}\right), 7.44$ 7.40 (m, 3 H), 7.39 - 7.33 (m, 2 H), 3.01 (t, $J=7.2, \mathrm{~Hz}, 2$ H), 2.92 (t, $J=7.2, \mathrm{~Hz}, 2 \mathrm{H}$ ), 2.50 (s, $3 \mathrm{H}), 2.08-2.04$ (m, $2 \mathrm{H}) ;{ }^{13} \mathrm{C}$ NMR (100 MHz, $\left.\mathrm{CDCl}_{3}\right): \delta 152.5,150.8,146.3$, 138.0, 138.0, 131.7, 128.5, 128.4, 127.3, 32.7, 30.9, 24.6, 21.9; IR (KBr): 3048, 3028, 2954, 2912, 2897, $1551(\mathrm{C}=\mathrm{N}) \mathrm{cm}^{-1}$; HRMS calcd for $\mathrm{C}_{15} \mathrm{H}_{15} \mathrm{~N}$ 209.1205, found 209.1207. 
5,6,7,8-Tetrahydro-1,4-dimethyl-3-phenylisoquinoline (3m).

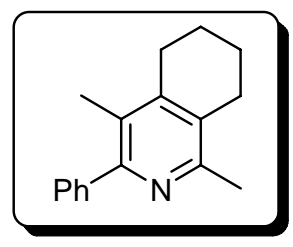

Yellow viscous oil; ${ }^{1} \mathrm{H}$ NMR (400 MHz, $\left.\mathrm{CDCl}_{3}\right)$ : $\delta 7.44$ - 7.39 (m, $\left.4 \mathrm{H}\right), 7.38$ 7.32 (m, 1 H), 2.65 - 2.64 (m, 4 H), 2.46 (s, 3 H), 2.11 (s, 3 H), 1.83 - 1.82 (m, 4 H); ${ }^{13} \mathrm{C}$ NMR (100 MHz, $\left.\mathrm{CDCl}_{3}\right): \delta 154.8,151.2,144.0,139.1,134.0,129.0,128.5$, 127.8, 126.9, 28.3, 26.2, 23.0, 22.5, 22.2, 22.1; IR (KBr): 3053, 2938, 2904, 2897, $1518(\mathrm{C}=\mathrm{N}) \mathrm{cm}^{-1}$; HRMS calcd for $\mathrm{C}_{17} \mathrm{H}_{19} \mathrm{~N}$ 237.1517, found 237.1518.

5,6,7,8-Tetrahydro-1,3-dimethyl-4-phenylisoquinoline (3m’).

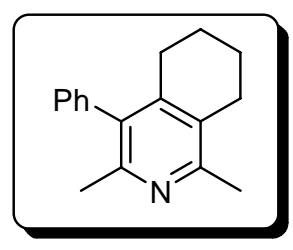

Yellow viscous oil; ${ }^{1} \mathrm{H}$ NMR (400 MHz, $\left.\mathrm{CDCl}_{3}\right): \delta 7.39$ (t, $\left.J=7.2 \mathrm{~Hz}, 2 \mathrm{H}\right)$, 7.32 (t, $J=7.2 \mathrm{~Hz}, 1 \mathrm{H}), 7.07$ (d, $J=7.2 \mathrm{~Hz}, 2 \mathrm{H}), 2.62$ (t, $J=6.4 \mathrm{~Hz}, 2 \mathrm{H}), 2.43$ (s, 3 H), 2.62 (t, J = 6.4 Hz, 2 H), 2.11 (s, $3 \mathrm{H}), 1.83$ - 1.82 (m, $4 \mathrm{H}) ;{ }^{13} \mathrm{C}$ NMR (100 MHz, $\left.\mathrm{CDCl}_{3}\right): \delta 154.6,153.4,145.0,141.5,129.1,129.0,128.0,127.1,126.4,27.2,26.4$ 22.3, 22.2, 22.1, 15.4; IR (KBr): 3042, 2983, 2910, 2839, $1536(\mathrm{C}=\mathrm{N}) \mathrm{cm}^{-1}$; HRMS calcd for $\mathrm{C}_{17} \mathrm{H}_{19} \mathrm{~N} 237.1517$, found 237.1520.

3-Ethyl-1-methyl-4-phenyl-5,6,7,8-tetrahydroisoquinoline (3n).

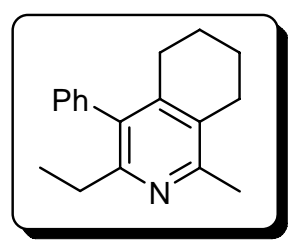

Yellow viscous oil; ${ }^{1} \mathrm{H}$ NMR (400 MHz, $\mathrm{CDCl}_{3}$ ): $\delta 7.43-7.33$ (m, $\left.3 \mathrm{H}\right), 7.13$ (d, $J=7.2 \mathrm{~Hz}, 2 \mathrm{H}), 2.65$ (t, $J=6.4 \mathrm{~Hz}, 2 \mathrm{H}$ ), 2.48 (s, $3 \mathrm{H}), 2.45$ (t, $J=7.6 \mathrm{~Hz}, 2 \mathrm{H}$ ), 
$1.79-1.75$ (m, $2 \mathrm{H}), 1.64-1.61$ (m, $2 \mathrm{H}), 1.08$ (t, $J=7.6 \mathrm{~Hz}, 3 \mathrm{H}) ;{ }^{13} \mathrm{C}$ NMR (100 $\left.\mathrm{MHz}, \mathrm{CDCl}_{3}\right): \delta 156.3,155.2,144.3,139.0,133.5,129.3,128.4,127.8,126.9,28.9$, 28.5, 26.3, 22.5, 22.3, 22.1, 14.6; IR (KBr): 3026, 2974, 2896, $1546(\mathrm{C}=\mathrm{N}) \mathrm{cm}^{-1}$; HRMS calcd for $\mathrm{C}_{18} \mathrm{H}_{21} \mathrm{~N} 251.1674$, found 251.1676.

\section{4-Ethyl-1-methyl-3-phenyl-5,6,7,8-tetrahydroisoquinoline (3n').}

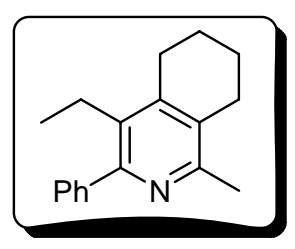

Product 3n' could not be separated from 3n by column chromatography. A copy of the ${ }^{1} \mathrm{H}$ NMR spectrum of the mixture is shown in page 26.

\section{Intermediate 8.}

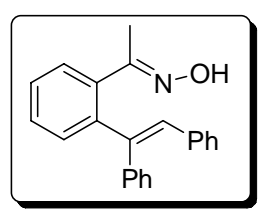

White solid; ${ }^{1} \mathrm{H}$ NMR (400 MHz, $\mathrm{CDCl}_{3}$ ): $\delta 8.36$ (s, $1 \mathrm{H}$ ), 7.35 - 7.26 (m, $4 \mathrm{H}$ ), 7.19 (d, $J=7.2 \mathrm{~Hz}, 1 \mathrm{H}), 7.18-7.08$ (m, $8 \mathrm{H}), 6.75$ (s, $1 \mathrm{H}), 1.86(\mathrm{~s}, 3 \mathrm{H}) ;{ }^{13} \mathrm{C} \mathrm{NMR}$ (125 MHz, $\left.\mathrm{CDCl}_{3}\right): \delta$ 158.6, 143.3, 141.8, 140.3, 137.5, 137.4, 131.0, 130.7, 130.5, 129.4, 129.0, 128.4, 128.0, 127.9, 127.5, 127.2, 126.8, 15.6; IR (KBr): $3566(\mathrm{OH})$, 3054, 3023, 2962, $1568(\mathrm{C}=\mathrm{N}) \mathrm{cm}^{-1}$; HRMS calcd for $\mathrm{C}_{22} \mathrm{H}_{19} \mathrm{NO} 313.1467$, found 313.1469.

\section{1-Methyl-3,4-diphenylisoquinoline (9).}

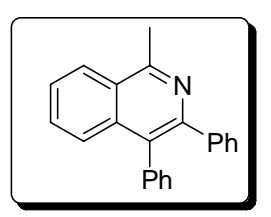

White solid; m.p. $174-176{ }^{\circ} \mathrm{C} ;{ }^{1} \mathrm{H}$ NMR (400 MHz, $\mathrm{CDCl}_{3}$ ): $\delta 8.20-8.18$ (m, 1 H), 7.65 - 7.56 (m, 3 H), 7.37-7.16 (m, $10 \mathrm{H}), 3.06$ (s, $3 \mathrm{H}) ;{ }^{13} \mathrm{C}$ NMR (100 MHz, 
$\left.\mathrm{CDCl}_{3}\right): \delta 157.7,149.4,141.0,137.5,136.0,131.3,130.2,129.9,129.1,128.1,127.5$ 127.0, 126.9, 126.5, 126.2, 126.1, 125.5, 22.7; IR (KBr): 3046, 2968, 1519 (C=N), $\mathrm{cm}^{-1}$; HRMS calcd for $\mathrm{C}_{22} \mathrm{H}_{17} \mathrm{~N} 295.1361$, found 295.1360 .

\section{NOE data.}

The structures of products $3 \mathbf{l}, \mathbf{3 m}$ 'and $3 \mathbf{n}$ were established by ${ }^{1} \mathrm{H}$ NMR NOE experiments. For example, selective irradiation of the Haproton at $\delta 3.00$ of $\mathbf{3 l}$ caused $1.01 \%$ enhancement of the ortho protons $H_{b}$ of the phenyl ring at $\delta 7.40$. Selective irradiation of the $H_{c}$ proton at $\delta 2.95$ caused $0.70 \%$ enhancement of the methyl protons at $\delta$ 2.45. Similar results were obtained for 3m' and 3n. These NOE results strongly confirm the structures of $\mathbf{3 l ,} \mathbf{3 m}$ ' and $\mathbf{3 n}$.
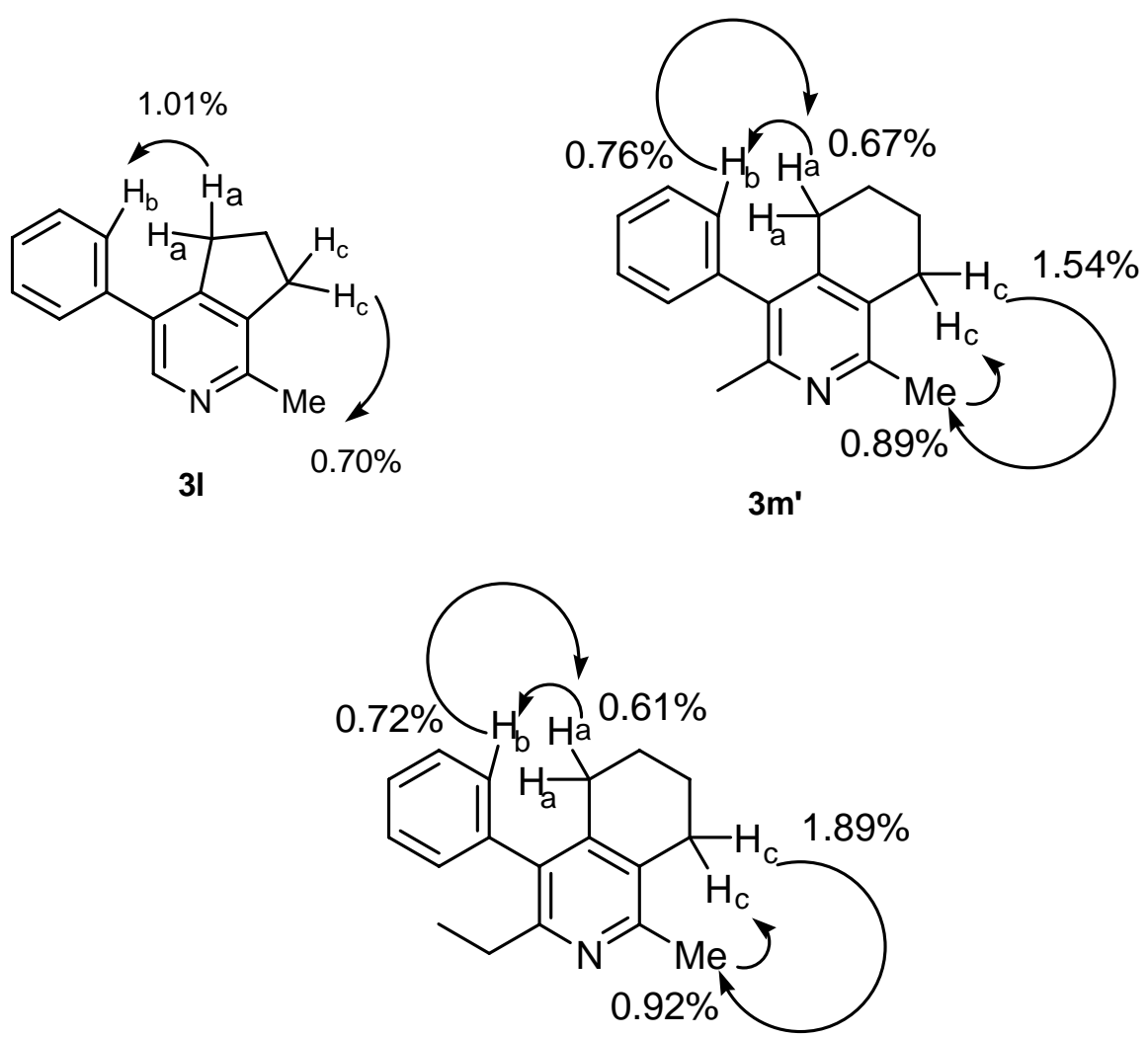

$3 n$ 


\section{References.}

1) Perrin, D. D.; Armarego, W. L. F. In Purification of Laboratory Chemicals, 3rd ed.; Pergamon Press: New York, 1988.

2) Mio, M. J.; Kopel, L. C.; Braun, J. B.; Gadzikwa, T. L.; Hull, K. L.; Brisbois, R. G.; Markworth, C. J.; Grieco, P. A. Org. Lett., 2002, 4, 3199.

3) (a) Shinada, T.; Yoshihara, K. Tetrahedron Lett., 1995, 36, 6701. (b) Booth, S. E.; Jenkins, P. R.; Swain, C. J.; Sweeney, J. B. J. Chem. Soc., Perkin Trans. 1 1994, 3499.

4) Osborn, J. A.; Wilkinson, G. In Reagents for Transition Metal Complex and Organometallic Syntheses; Angelici, R., Ed.; Wiley: New York, 1989; Vol. 28, pp. $77-79$ 
${ }^{1} \mathrm{H}$ and ${ }^{13} \mathrm{C}$ NMR spectra of compound 3a.
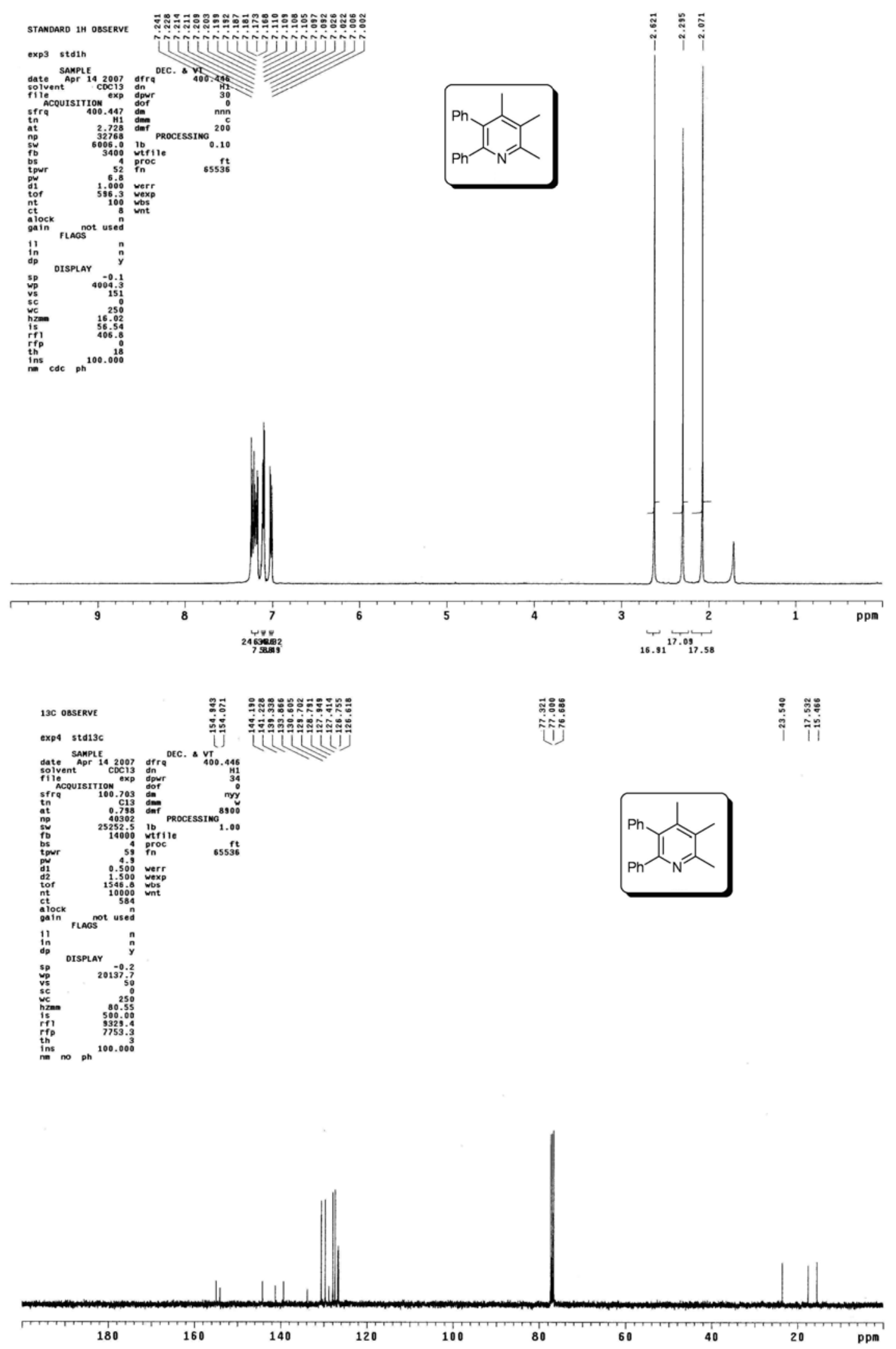
${ }^{1} \mathrm{H}$ and ${ }^{13} \mathrm{C}$ NMR spectra of compound $\mathbf{3 b}$
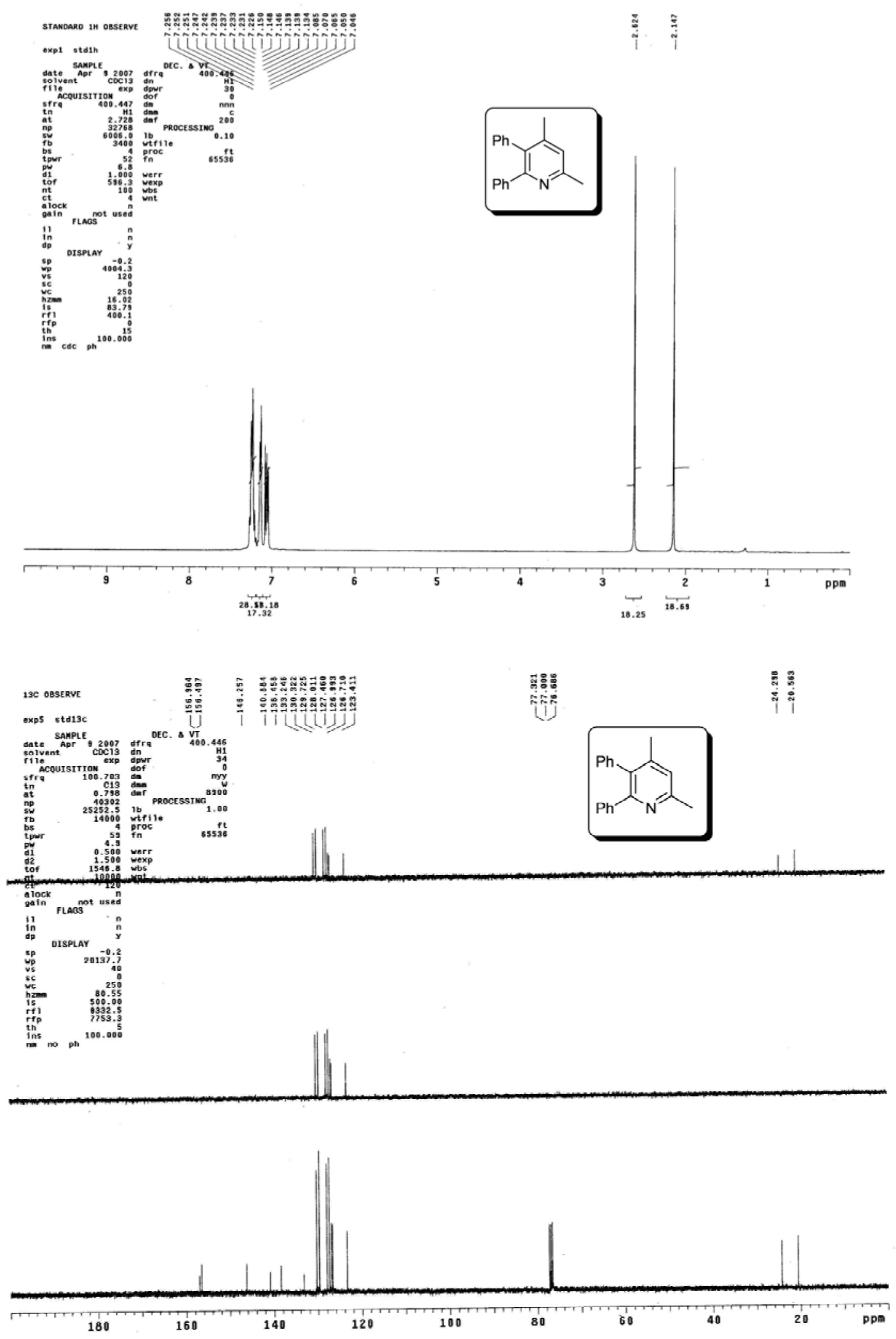
${ }^{1} \mathrm{H}$ and ${ }^{13} \mathrm{C}$ NMR spectra of compound 3c
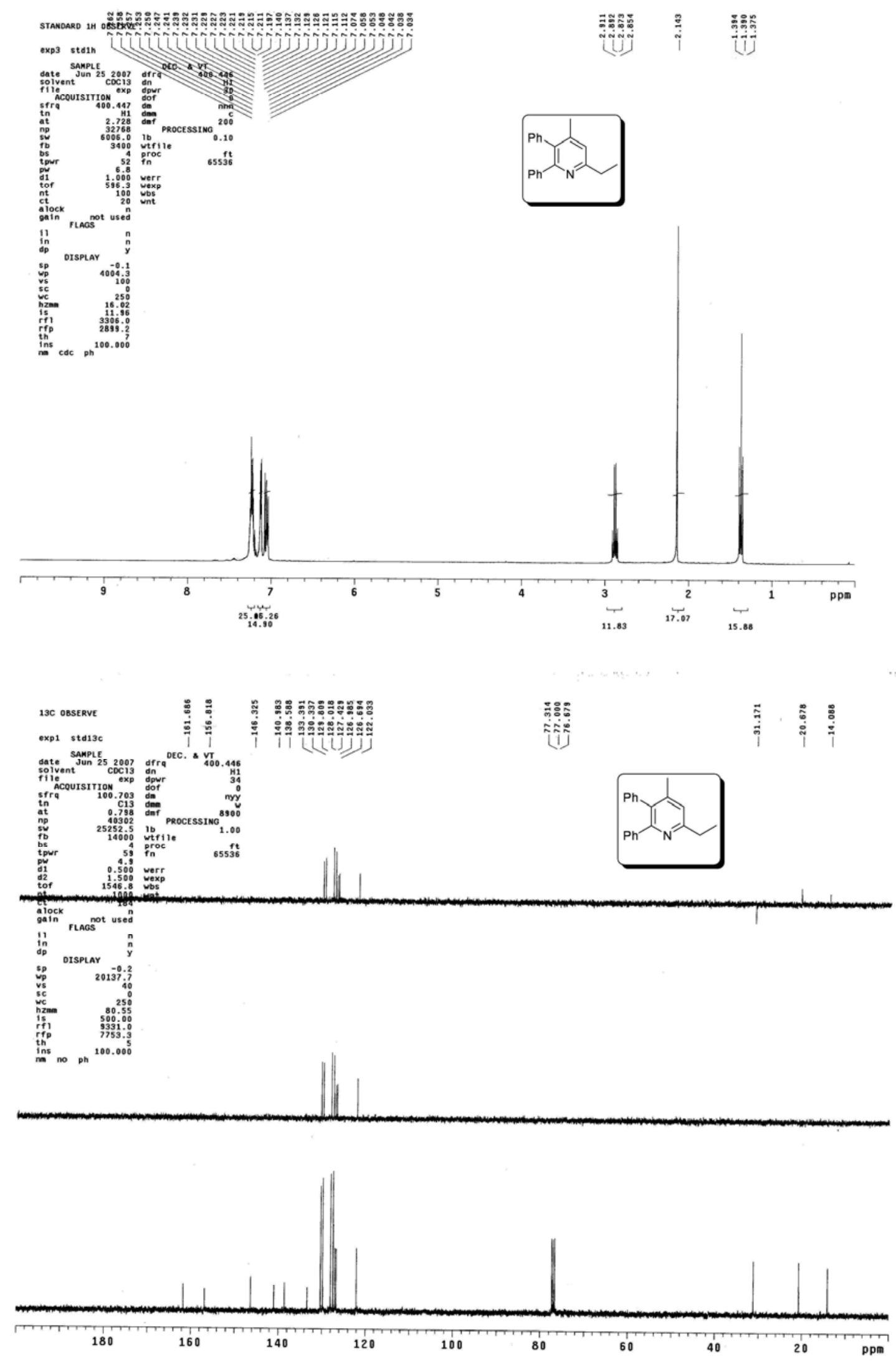
${ }^{1} \mathrm{H}$ and ${ }^{13} \mathrm{C}$ NMR spectra of compound 3d
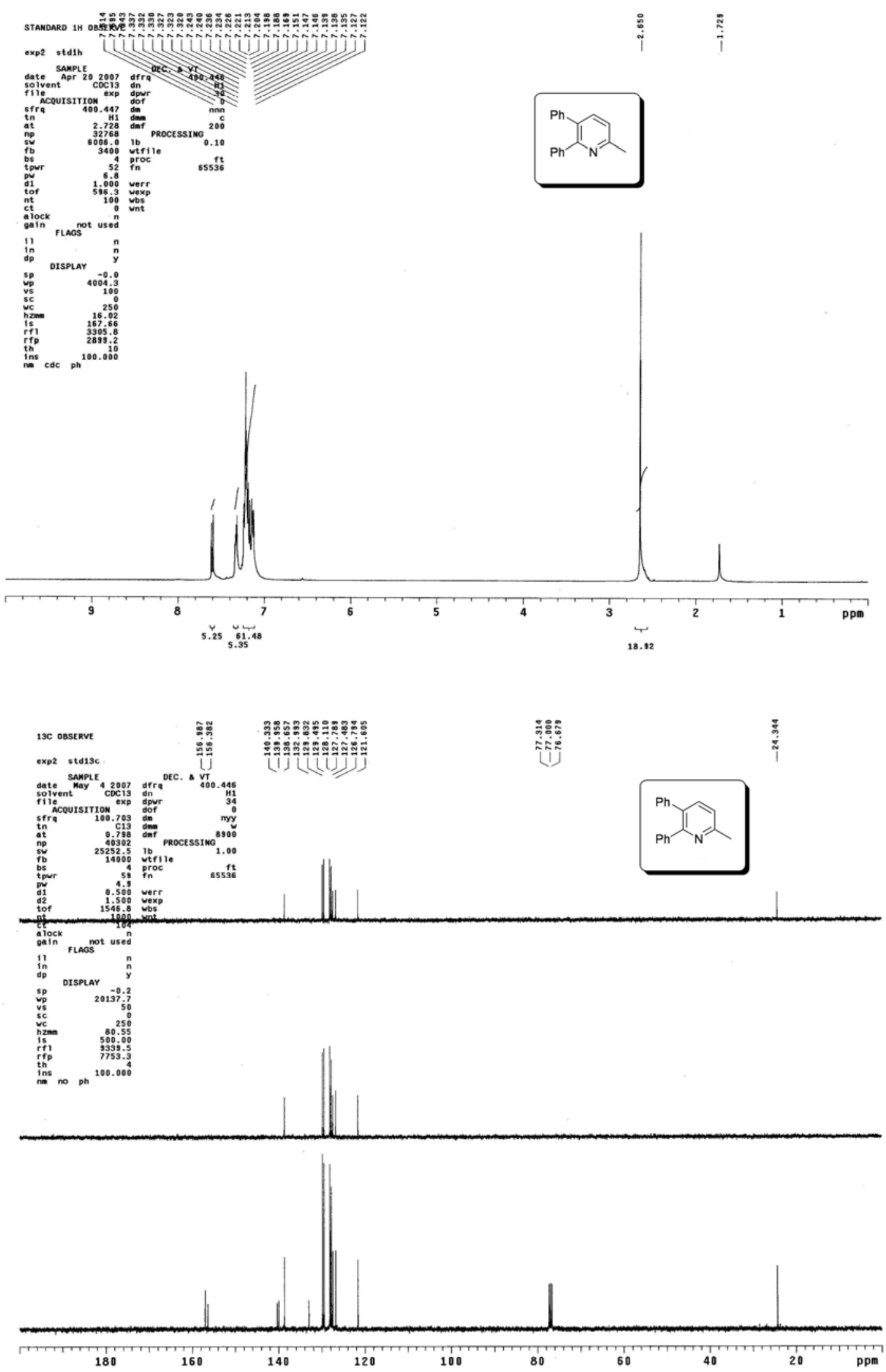
${ }^{1} \mathrm{H}$ and ${ }^{13} \mathrm{C}$ NMR spectra of compound $\mathbf{3 e}$
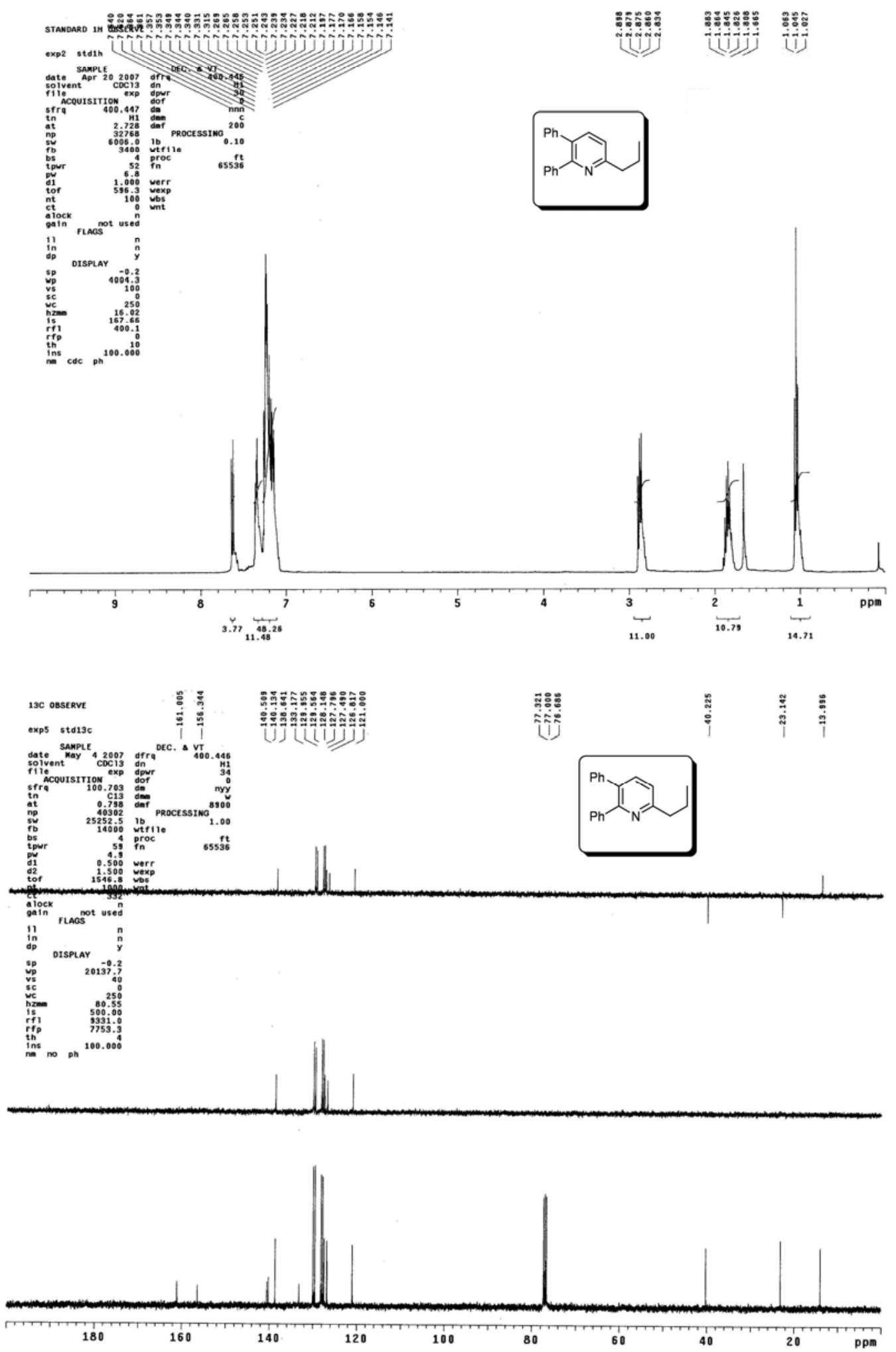
${ }^{1} \mathrm{H}$ and ${ }^{13} \mathrm{C}$ NMR spectra of compound $3 \mathbf{f}$.
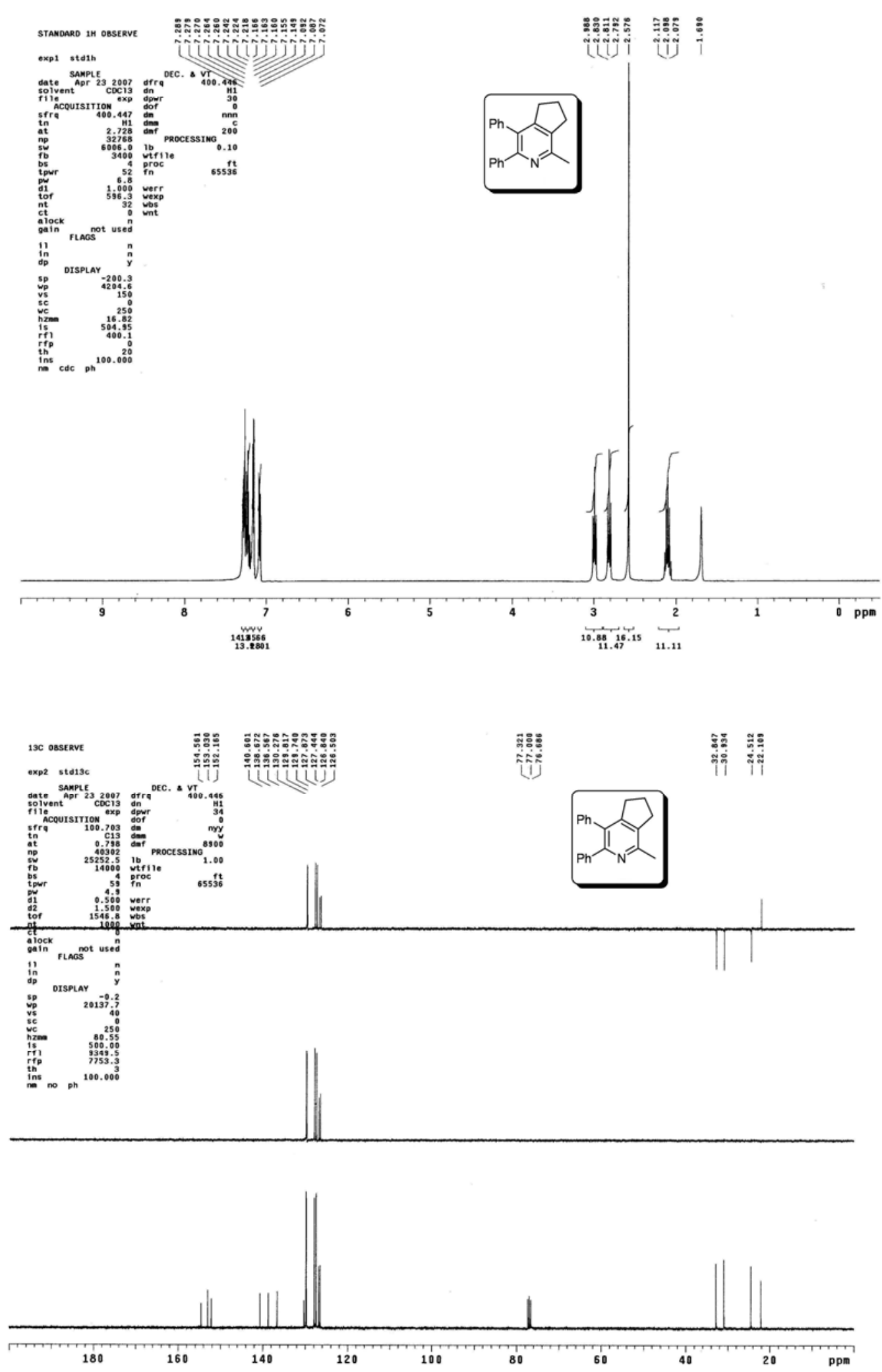
${ }^{1} \mathrm{H}$ and ${ }^{13} \mathrm{C}$ NMR spectra of compound $3 g$.
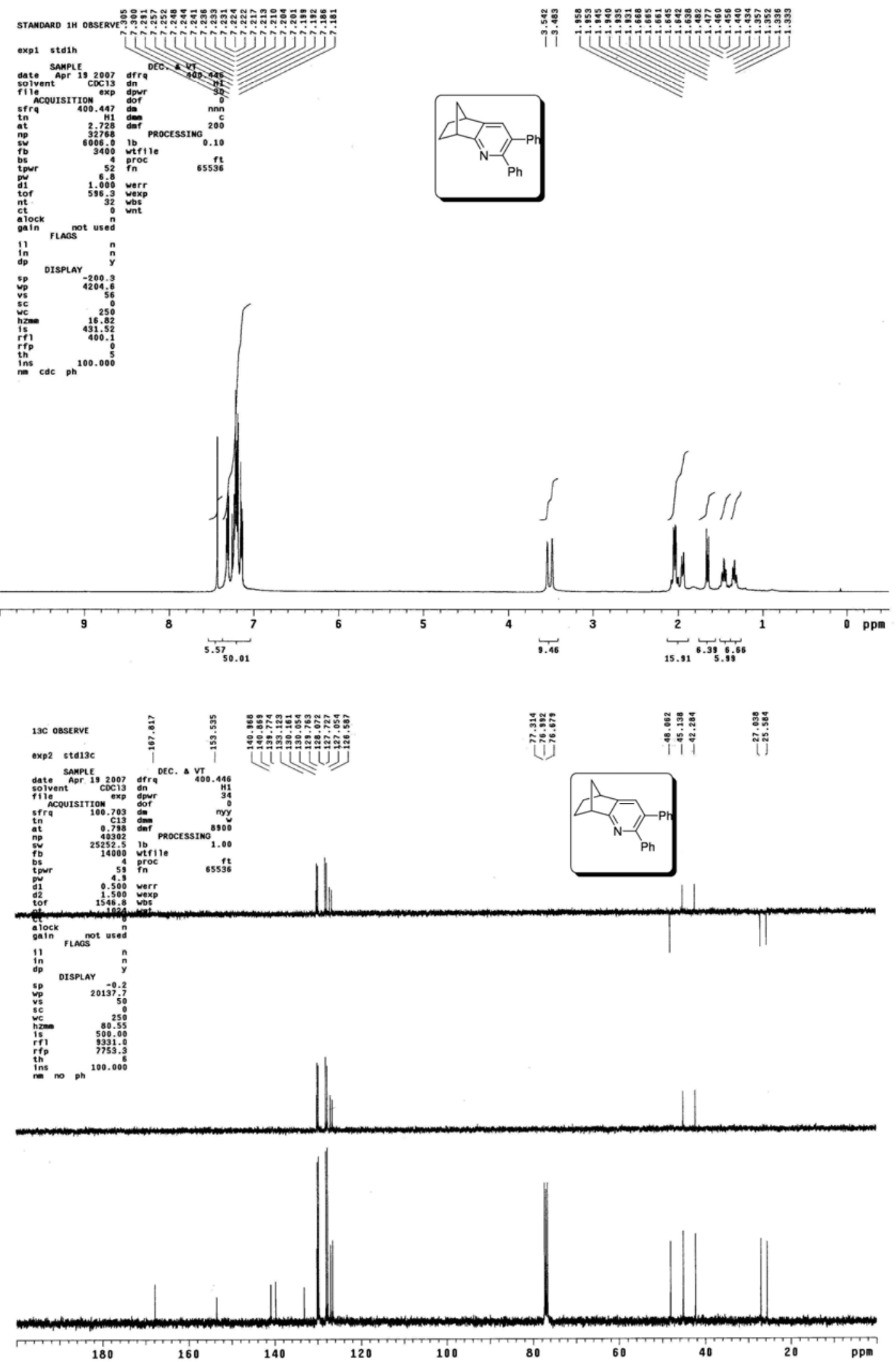
${ }^{1} \mathrm{H}$ and ${ }^{13} \mathrm{C}$ NMR spectra of compound $\mathbf{3 h}$.

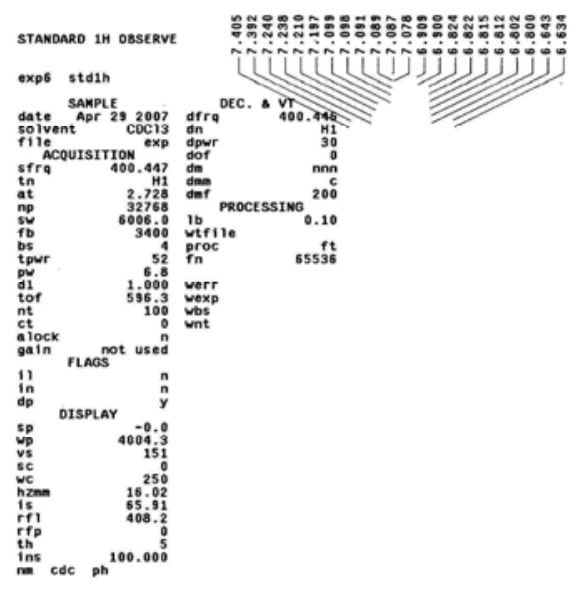

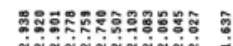

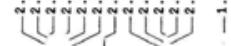
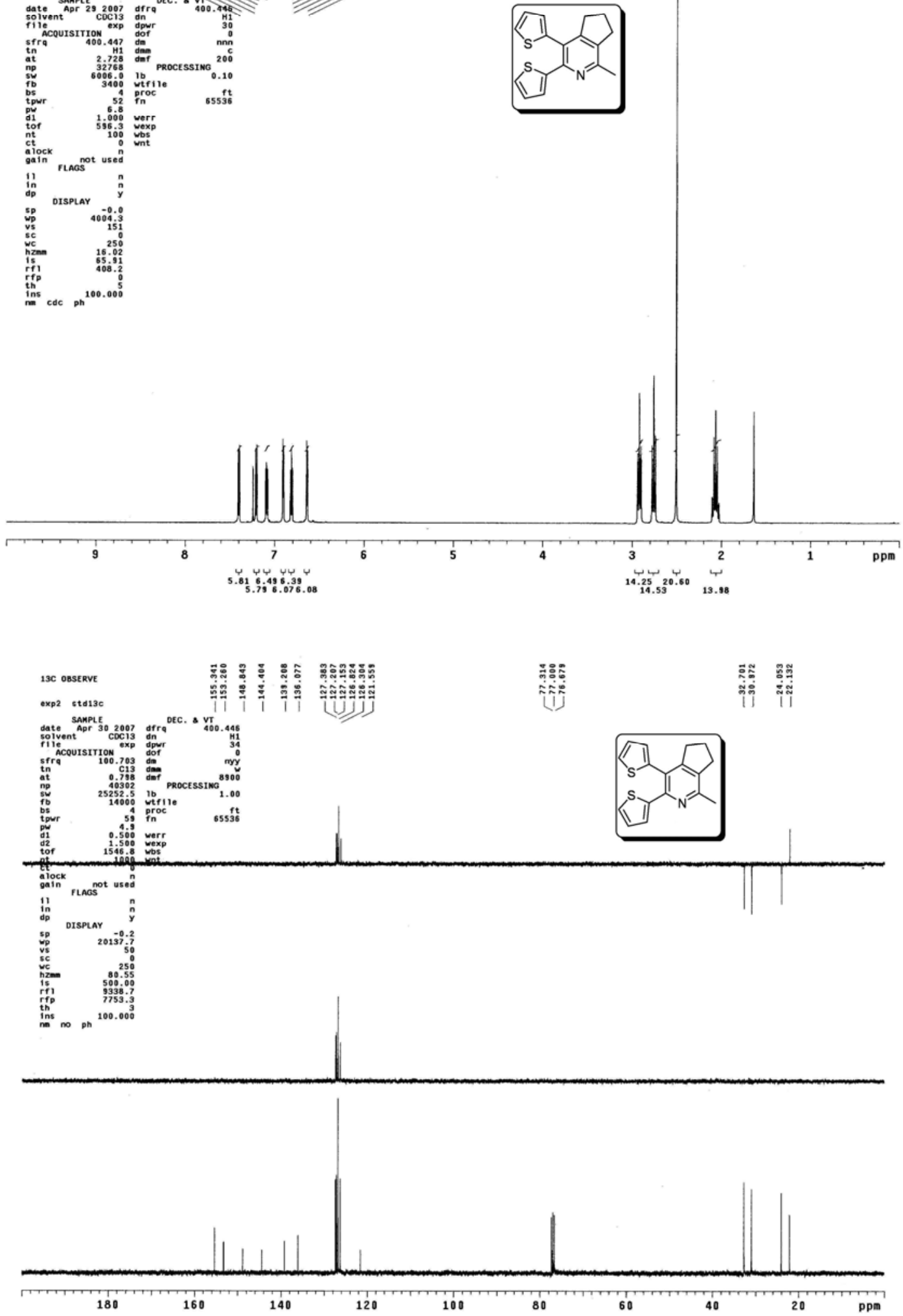
${ }^{1} \mathrm{H}$ and ${ }^{13} \mathrm{C}$ NMR spectra of compound $3 \mathbf{i}$.
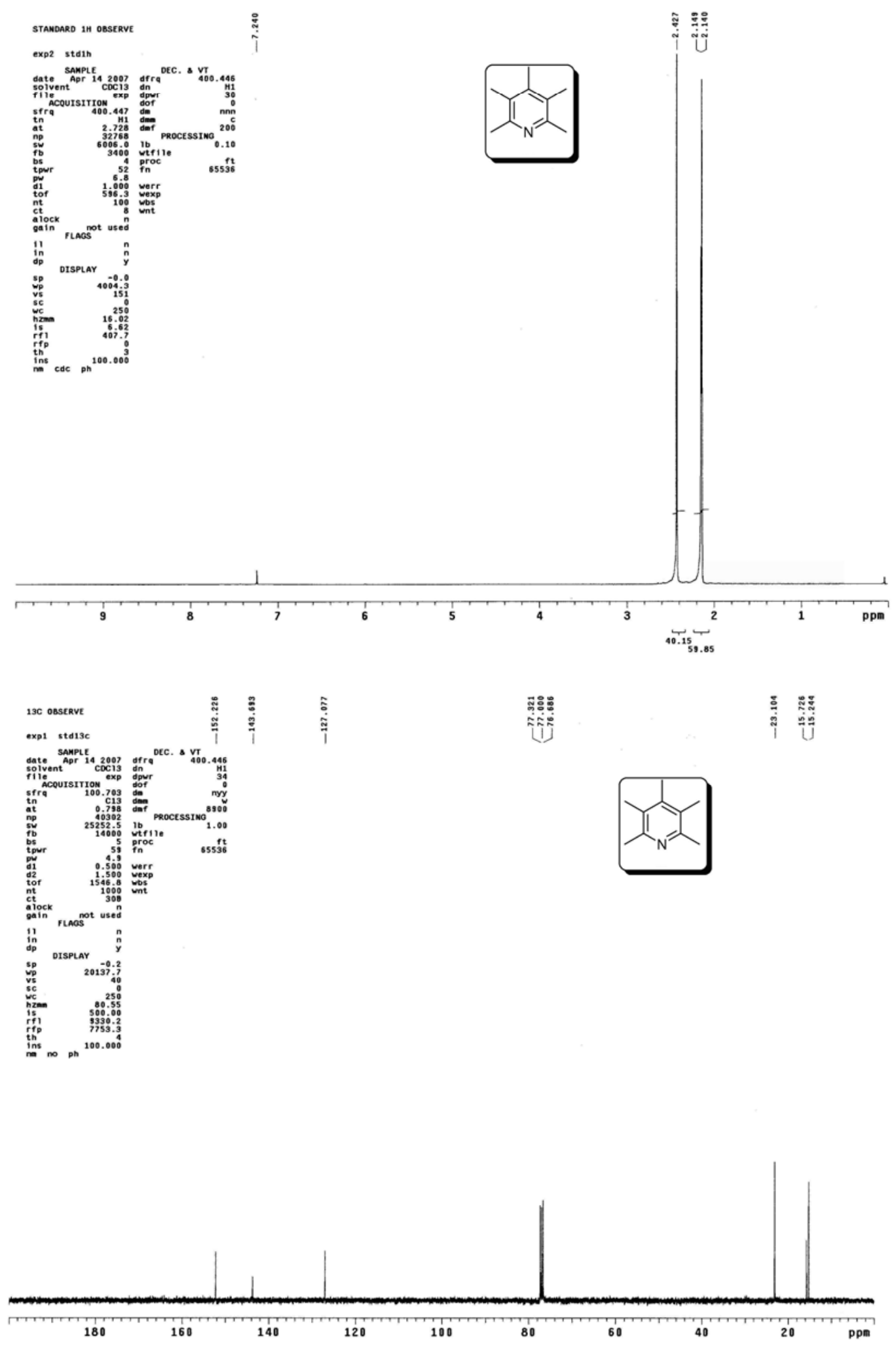
${ }^{1} \mathrm{H}$ and ${ }^{13} \mathrm{C}$ NMR spectra of compound $\mathbf{3 j}$.
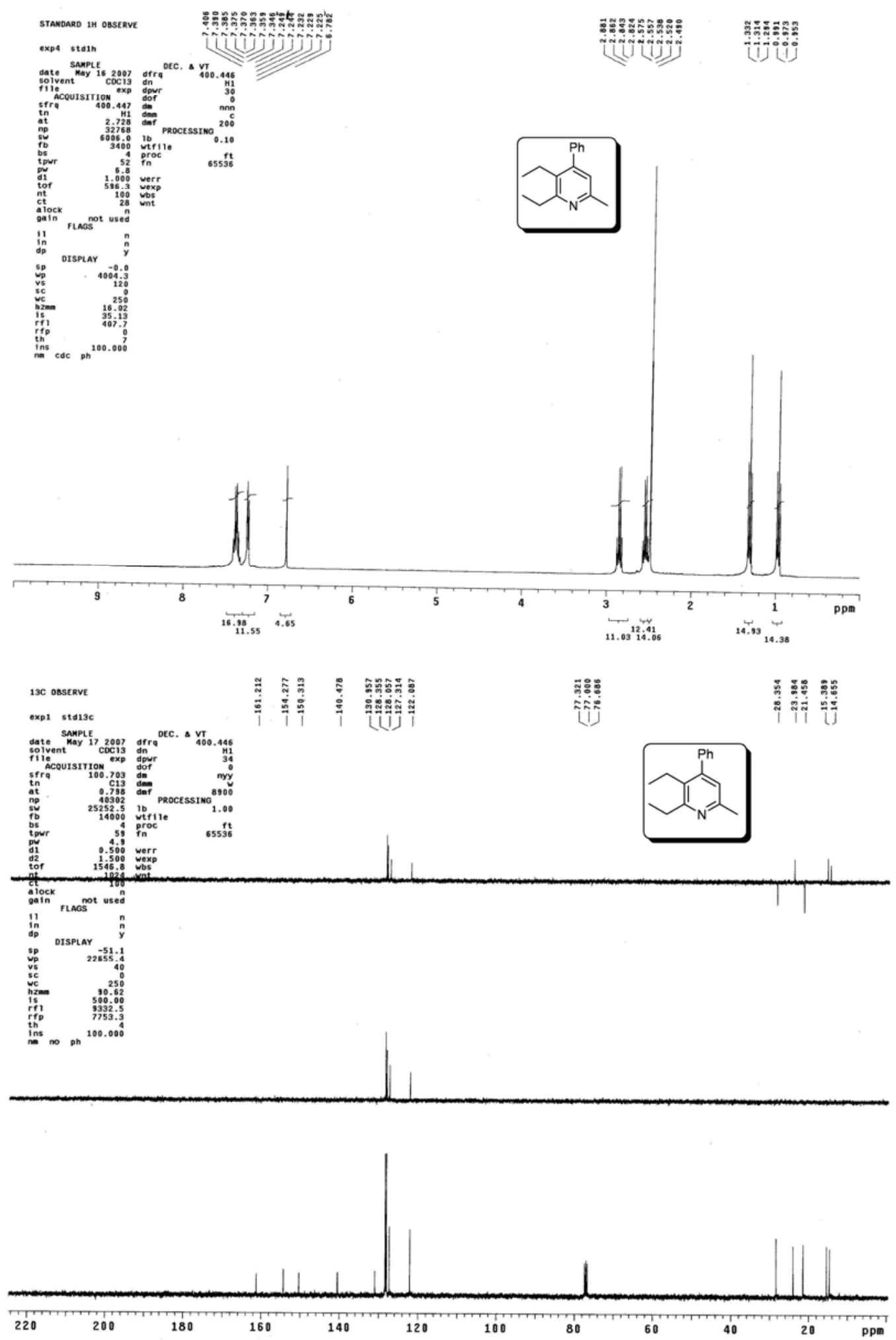
${ }^{1} \mathrm{H}$ and ${ }^{13} \mathrm{C}$ NMR spectra of compound 3k.
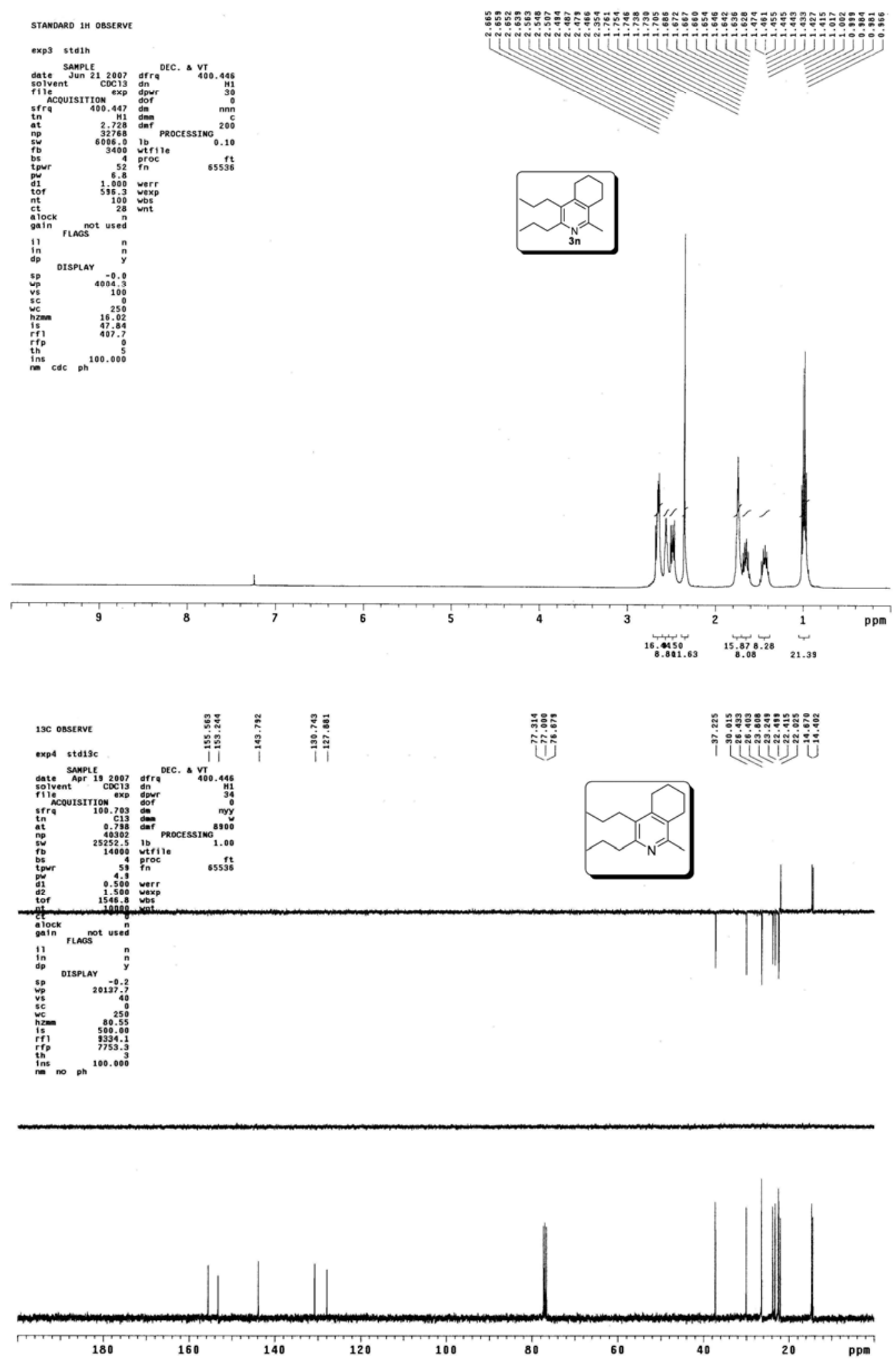

${ }^{1} \mathrm{H}$ and ${ }^{13} \mathrm{C}$ NMR spectra of compound 31. 

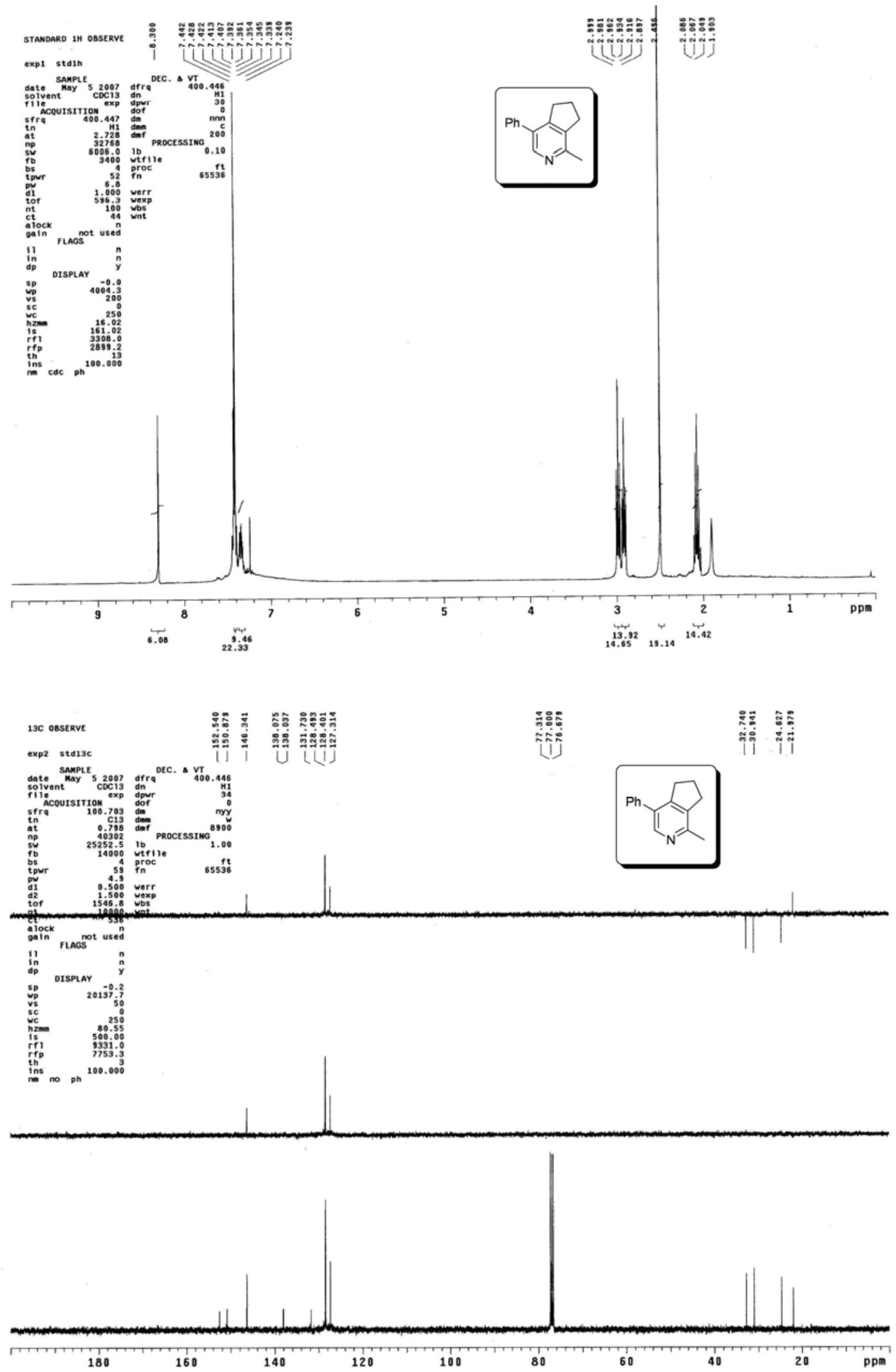
${ }^{1} \mathrm{H}$ and ${ }^{13} \mathrm{C}$ NMR spectra of compound 3m
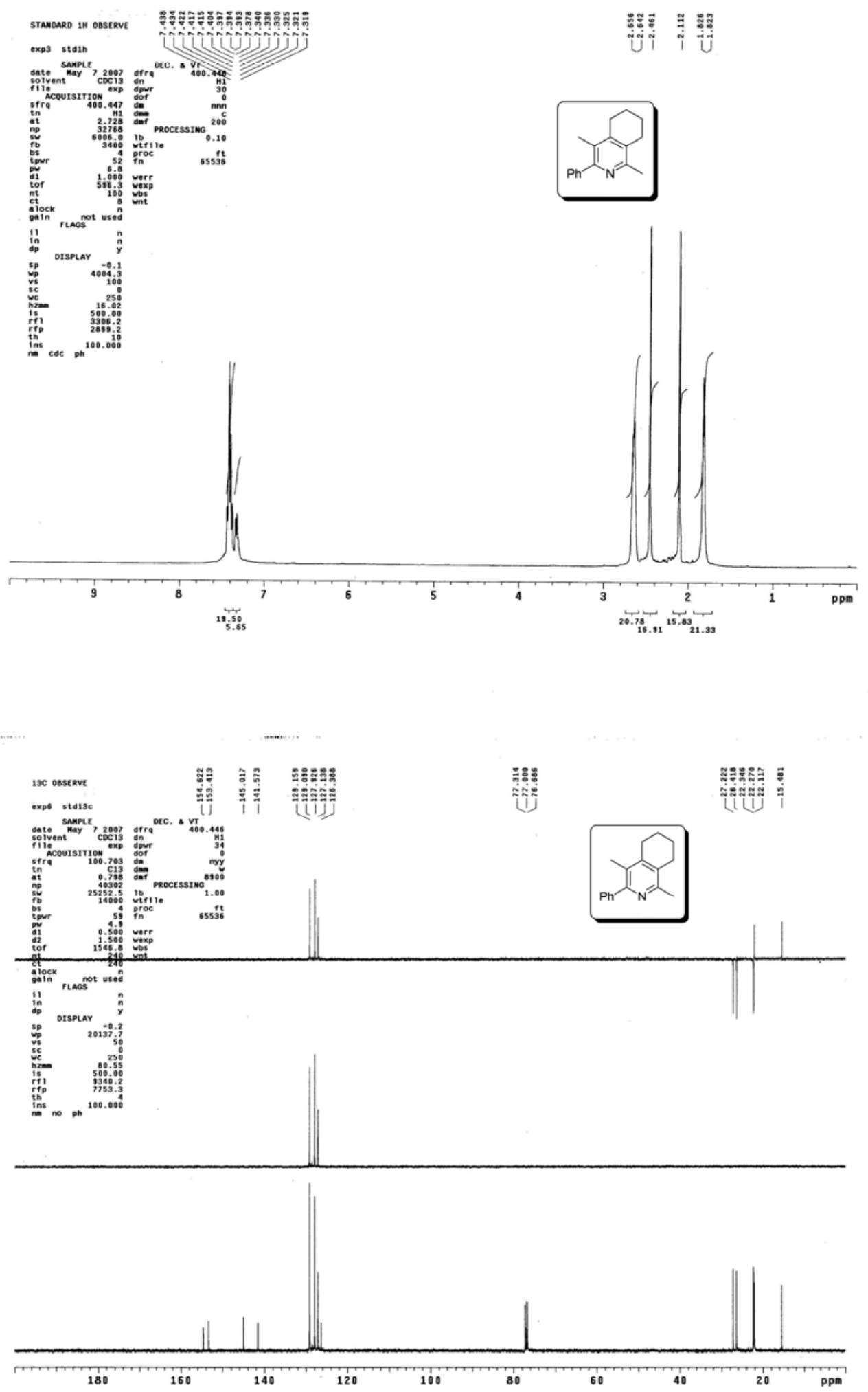
${ }^{1} \mathrm{H}$ and ${ }^{13} \mathrm{C}$ NMR spectra of compound 3m'
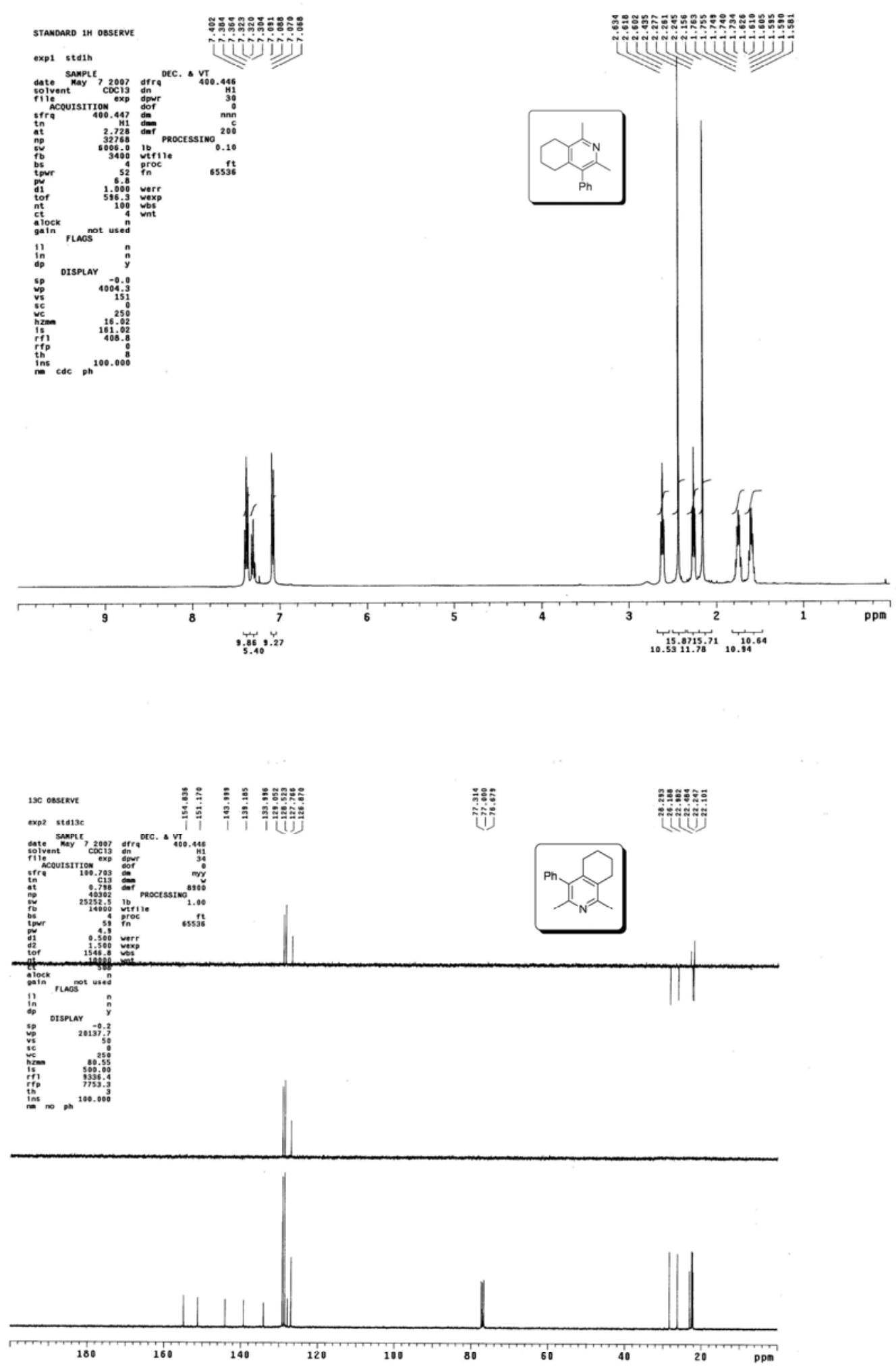
${ }^{1} \mathrm{H}$ and ${ }^{13} \mathrm{C}$ NMR spectra of compound $\mathbf{3 n}$.

4

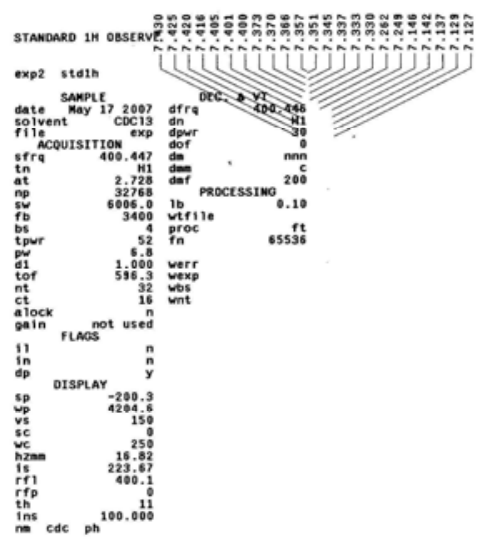

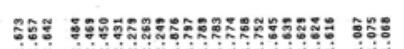
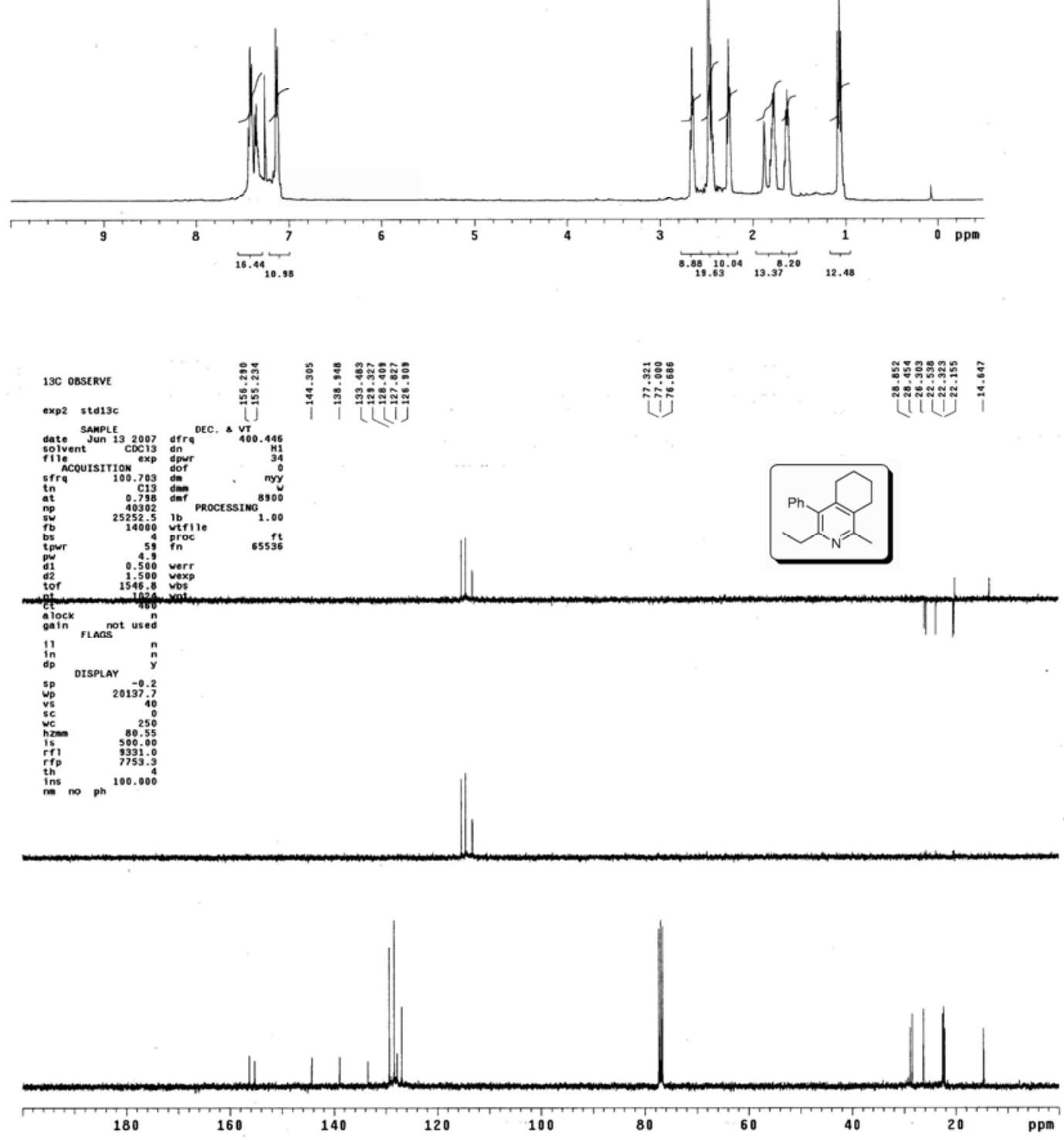
Mixture of ${ }^{1} \mathrm{H}$ NMR spectrum of $3 \mathbf{n}$ and 3n'.
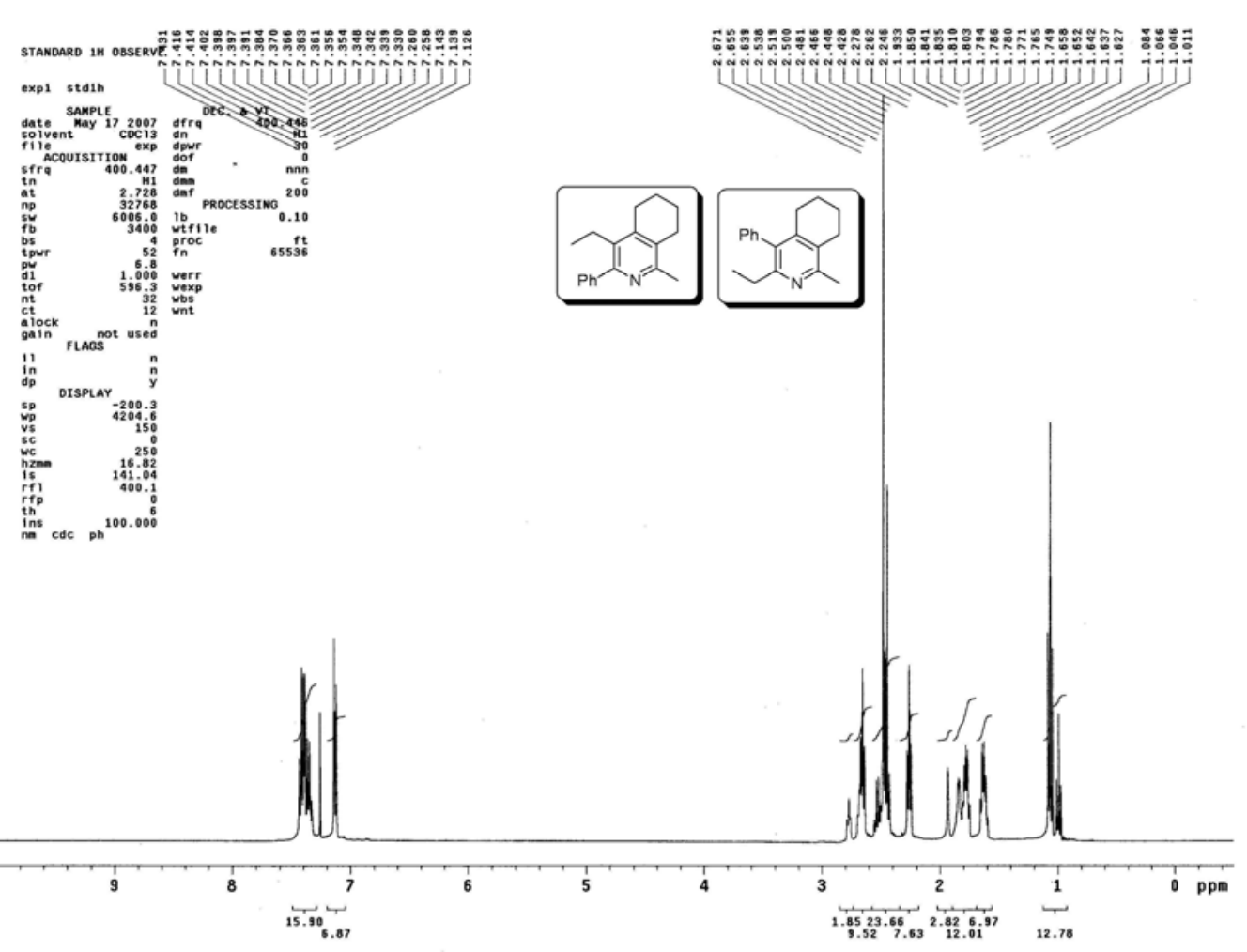
${ }^{1} \mathrm{H}$ and ${ }^{13} \mathrm{C}$ NMR spectra of intermediate 8 .
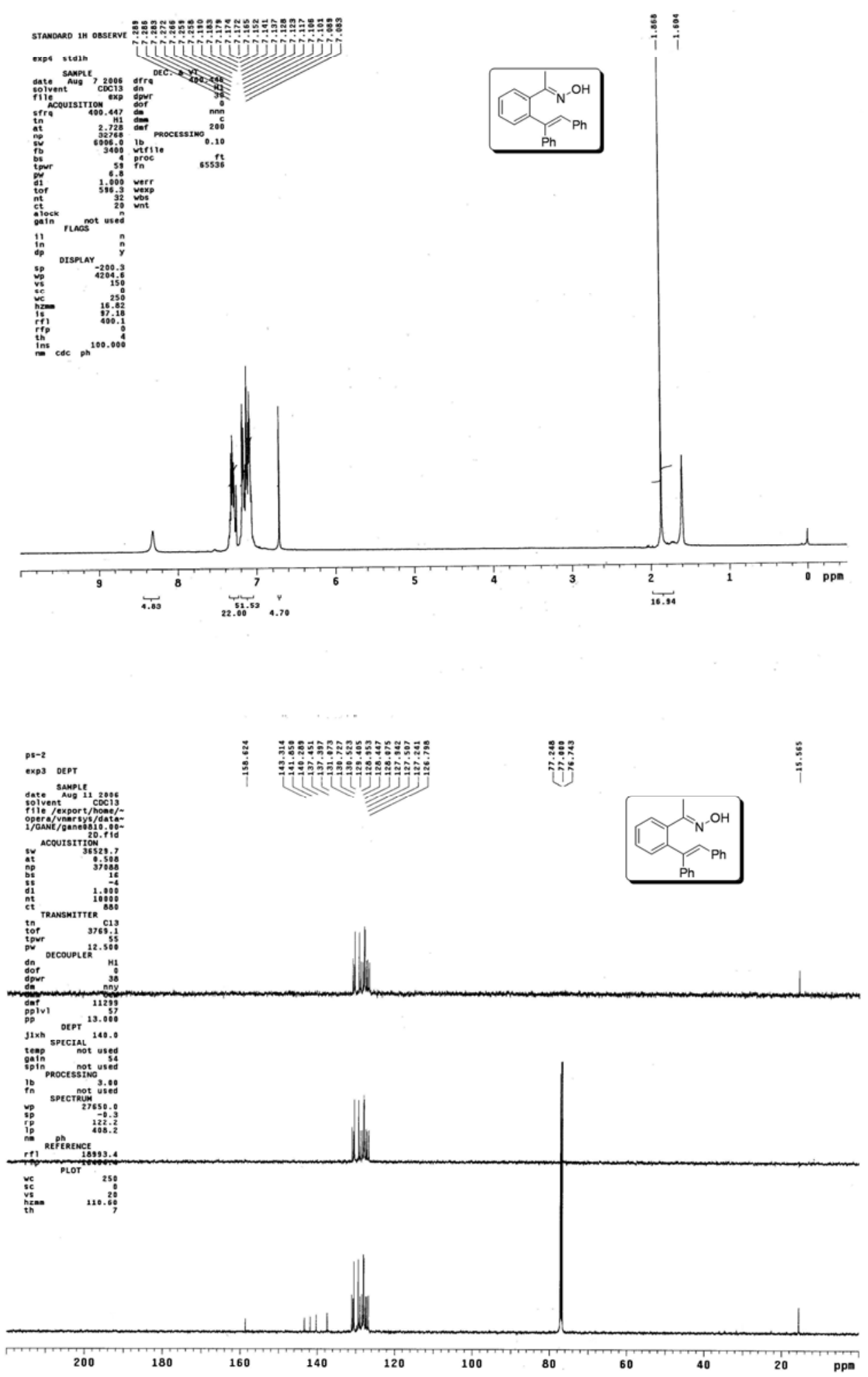
${ }^{1} \mathrm{H}$ and ${ }^{13} \mathrm{C}$ NMR spectra of compound $\mathbf{9}$.
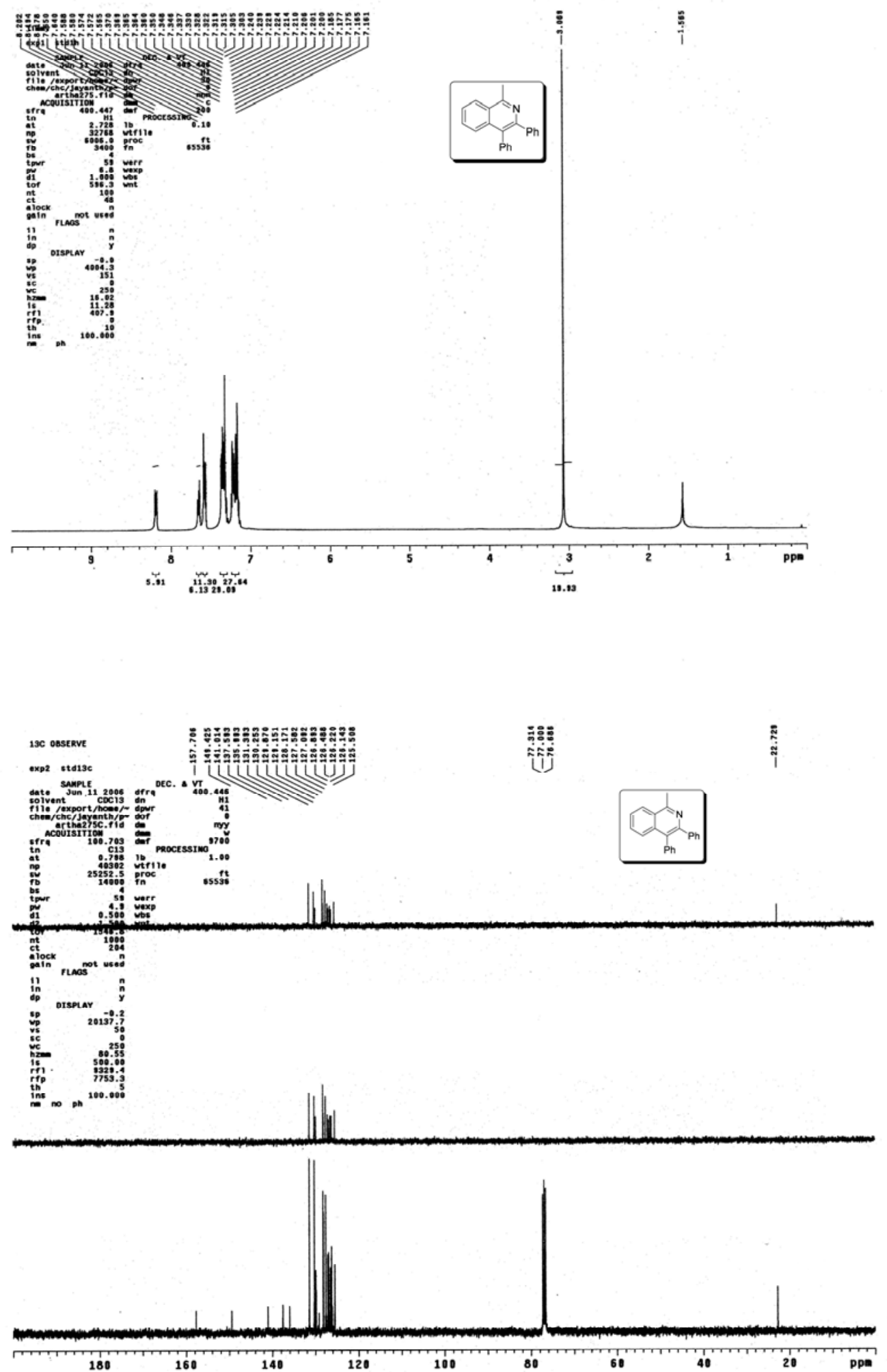
Expansion of ${ }^{13} \mathrm{C}$ NMR spectrum of compound $\mathbf{9}$
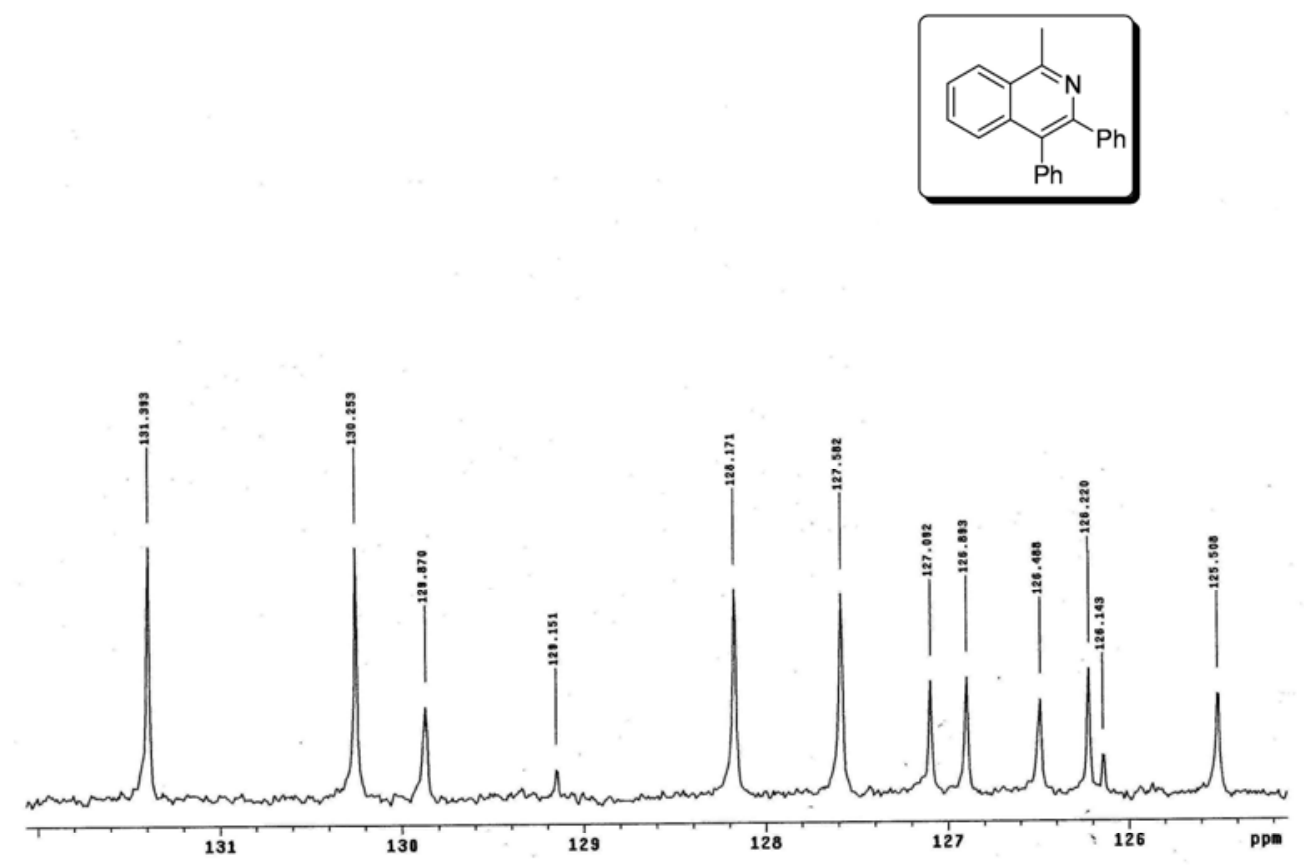